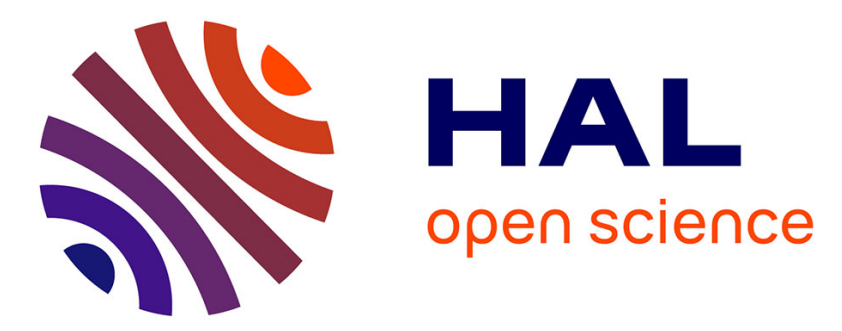

\title{
Combining rights and welfarism: a new approach to intertemporal evaluation of social alternatives
}

\author{
Ngo van Long, Vincent Martinet
}

\section{To cite this version:}

Ngo van Long, Vincent Martinet. Combining rights and welfarism: a new approach to intertemporal evaluation of social alternatives. Social Choice and Welfare, 2018, 50 (1), pp.35-64. 10.1007/s00355017-1073-5 . hal-01634283

\section{HAL Id: hal-01634283 \\ https://hal.science/hal-01634283}

Submitted on 13 Nov 2017

HAL is a multi-disciplinary open access archive for the deposit and dissemination of scientific research documents, whether they are published or not. The documents may come from teaching and research institutions in France or abroad, or from public or private research centers.
L'archive ouverte pluridisciplinaire HAL, est destinée au dépôt et à la diffusion de documents scientifiques de niveau recherche, publiés ou non, émanant des établissements d'enseignement et de recherche français ou étrangers, des laboratoires publics ou privés. 


\title{
Combining Rights and Welfarism: a new approach to intertemporal evaluation of social alternatives
}

\author{
Ngo Van Long*and Vincent Martinet ${ }^{\dagger}$
}

October 3, 2017

Acknowledgments: We thank the participants of the CIREQ Natural Resource Economics workshop (Montreal, January 2012, December 2015), IFO lunch time seminar (Munich, January, 2012), SURED 2012 (Ascona), EAERE 2012 (Prague), Ecole Polytechnique seminar (Palaiseau, December 2012), and CER-ETH Zürich Research Seminar (November 2015). We thank Bob Cairns, Hassan Benchekroun, Hans-Werner Sinn, Karen Pittel, Antoine Bommier, Hans Gersbach, Lucas Bretschger, Julien Daubanes as well as the referees and the editor for comments.

*Department of Economics, McGill University, Montreal H3A 2T7, Canada, and Tasmanian School of Business, University of Tasmania.

E-mail: ngo.long@mcgill.ca, phone: +1-514-398-4844, fax: +1-514-398-4938.

$\dagger$ Economie publique, AgroParisTech, INRA, Université Paris-Saclay, 78850, Thiverval-Grignon, France. E-mail: vincent.martinet@inra.fr, phone: +33-1-3081-5357, fax: +33-1-3081-5368. 


\title{
Combining Rights and Welfarism: a new approach to intertemporal evaluation of social alternatives
}

\begin{abstract}
We propose a new criterion reflecting both the concern for rights and the concern for welfare in the evaluation of economic development paths. The concern for rights is captured by a pre-ordering over combinations of thresholds corresponding to floors or ceilings on various quantitative indicators. The resulting constraints on actions and on levels of state variables are interpreted as minimal rights to be guaranteed to all generations, for intergenerational equity purposes. The level of these rights are endogenously chosen, accounting for the "cost in terms of welfare" of granting them. Such a criterion could embody the idea of sustainable development. We provide an axiomatization of such a criterion and characterize the tension between rights and welfare in a general economic framework. We apply the criterion to the standard Dasgupta-Heal-Solow model of resource extraction and capital accumulation. We show that if the weight given to rights in the criterion is sufficiently high, the optimal solution is on the threshold possibility frontier. The development path is then "driven" by the rights. In particular, if a minimal consumption is considered as a right, constant consumption can be optimal even with a positive utility discount rate. In this case, the shadow value of the right plays an important role in the determination of the rate of discount to be applied to social investment projects.
\end{abstract}

JEL-Classifications: D63, H43, Q01.

Keywords: Rights, Welfare, Intergenerational Equity, Sustainability. 


\section{Introduction}

Much of normative economic theory is built on the premises that individuals seek to maximize their utility and that social welfare is the (weighted) sum of individual utilities. Under utilitarianism, or more generally welfarism, it is legitimate to prescribe policies that lead to increase the utility of some individuals at the expense of that of other individuals, as long as social welfare rises. At some extreme, the life of a person could be sacrificed for "the greater good" of the society. In an intergenerational context, the welfare of a generation could be sacrificed without limits to increase intertemporal welfare by raising the welfare of other generations. Many philosophers have expressed the concern that welfarism does not take rights seriously. They argue that all individuals should be entitled to some basic rights, such as life, health, and a "decent standard of living." Rawls (1971) pointed out that "optimal growth" (under some utilitarian objectives) may unreasonably require too much savings from poor generations for the benefits of their wealthier descendants. More recently, the same rationale has led environmentalists to argue that the present generations, in their pursuit of wealth and well-being, are depriving future generations of their rights to natural assets.

Sustainable development has been described in the Brundtland report (WCED, 1987) as development "that meets the needs of the present without compromising the ability of future generations to meet their own needs." Current growth patterns induce concerns for sustainability, in particular with respect to environmental degradations. Intergenerational equity and environmental issues are cornerstones of sustainability. Sustainability may require to add constraints to the intertemporal optimization of the distribution of welfare between present and future people for deontological (or "Kantian") ethical reasons (Howarth, 1995). Along these lines, it is argued that society should impose constraints, in the form of floors or ceilings, on various variables of the development path. Reflecting the concerns for rights, environmental issues are often addressed by setting objectives in terms of quantitative thresholds which should not be overshot. For example biodiversity should not fall below a certain level, while emissions of pollutants should not exceed a certain level. Socio-economic thresholds could also be mentioned, for instance on health and education. These thresholds can be interpreted as minimal rights to be guaranteed to all generations. Of course, if floors are too high and ceilings are too low, the set of possible actions will be empty. To rule out such a case, one has to address the trade-offs among minimal rights, as described in Martinet (2011) for the definition of sustainability objectives (i.e., quantities which should be sustained over time). Moreover, imposing a sustainability constraint on the growth path induces a cost in terms of welfare. In a 
different context, not referring to rights but to a concern for the utility of the worse-off generation, Alvarez-Cuadrado and Long (2009) showed that there is a trade-off between welfare and the sustained level of utility optimally chosen by the society. Rights could induce a welfare cost. In the climate change debate, a ceiling on green house gases concentration would impose restrictions on the current growth pattern as emissions would have to be curtailed. This is the cost of providing future generations the right to live in a more or less tolerable climate. When defining such an environmental constraint, current generations should trade off this cost against the value attributed to the level of the environmental objective they agree to sustain for future generations. There is a tension between rights and welfare, which has not been formally modeled in the literature.

In this paper, we put forward the view that society may not only seek to maximize welfare (in a standard sense offered by welfare economics), but may also be concerned with rights. Society thus makes trade-offs between rights and welfare. To represent these trade-offs, we propose a criterion for ranking social alternatives, based on an indicator called Rights and Welfare Indicator (RWI for short). This indicator combines an index of rights, such as the right to satisfy basic needs or the right to have access to some natural assets, with a welfare index based on the conventional utilitarian objective of maximizing the integral of the discounted stream of utility derived from the consumption of goods and services. The index of rights is an aggregate measure of various thresholds representing "sustainability" in a broad sense. The optimization problem endogenously determines the rights guaranteed to all generations, accounting for their cost in terms of welfare. The optimal, RWI-maximizing development path that satisfies these rights is also characterized, allowing us to describe the implications of these sustainability objectives on the path of resource use.

We show that, depending on the preferences and the relative weight accorded to minimal rights, the optimal development path may either be a constrained utilitarian path, or switch to a development path fully characterized by the minimal rights guaranteed to all generations (right-based sustainable development). We illustrate the general results by applying the criterion to the standard Dasgupta-Heal-Solow model of resource extraction and capital accumulation. In this model, we find that, when a minimal consumption constraint is effective, the implied social discount rate is different from that obtained under the classical utilitarian formulation.

The remaining of the paper is organized as follows. The motivation of our approach is detailed in Section 2. We present therein the tension between rights and welfare, as well as a brief history of sustainability criteria to put our criterion in perspective. The studied criterion is introduced in Section 3, providing both an axiomatization of 
the social choice of rights and welfare streams and a tractable criterion to be applied to economic growth models. Results on the trade-off between rights and welfare are given in Section 4, as well as a direct approach characterizing the optimal solution of the criterion maximization (optimal minimal rights and optimal development path) in a general economic model. The results are illustrated in the Dasgupta-Heal-Solow model of nonrenewable resource depletion and capital accumulation in Section 5. We provide concluding remarks in Section 6.

\section{Motivation}

\subsection{Rights versus Welfare}

The tension between rights and welfarist considerations has long been a subject of debate among philosophers, thinkers and economists. The Rawlsian theory of justice places rights above welfare. Rawls' conception of justice has its foundation in the theory of social contract advanced by Locke, Rousseau, and Kant. The initial position conceived by Rawls is a hypothetical situation in which the contracting parties are individuals hidden behind the veil of ignorance: none of them knows his place in society, his natural talents, intelligence, strength, and the like. In other words, the principles of justice are agreed to in an initial situation that is fair. Rawls' first principle of justice is that "each person is to have an equal right in the most extensive scheme of equal basic liberties compatible with a similar scheme of liberties for others." His second principle of justice, the difference principle, insists that social and economic inequalities (and in particular difference in income) are acceptable only if they are arranged so that they are "both (a) to the greatest expected benefit of the least advantaged and (b) attached to offices and positions open to all under conditions of fair equality of opportunity." 1

This concern extends to intergenerational equity, for example when optimal growth requires too much savings from poor generations for the benefits of their wealthier descendants. Immanuel Kant (1724-1804) found it disconcerting that earlier generations should carry the burdens for the benefits of later generations. In his essay, Idea for a Universal History with a Cosmopolitan Purpose, Kant put forward the view that nature

\footnotetext{
${ }^{1}$ Another influential philosopher who stressed the preponderance of rights is Nozick (1974). He emphasized the importance of property rights, from a somewhat different perspective. Nozick's work has inspired alternative articulations of libertarian rights with a game-theoretic flavor. See Gärdenfors (1981); Sugden (1985); Gaertner et al. (1992); Deb (1994); Hammond (1995, 1996); Peleg (1998); Suzumura and Yoshihara (2008), among others, and the overview proposed by Suzumura (2005). These papers acknowledge the fundamental contribution of Sen (1970a,b, 1976). In our paper, we abstract from gametheoretic considerations.
} 
is concerned with seeing that man should work his way onwards to make himself worthy of life and well-being: "What remains disconcerting about all this is firstly, that the earlier generations seem to perform their laborious tasks only for the sake of the later ones, so as to prepare for them a further stage from which they can raise still higher the structure intended by nature; and secondly, that only the later generations will in fact have the good fortune to inhabit the building on which a whole series of their forefathers ... had worked without being able to share in the happiness they were preparing "(see Reiss, 1970, p. 44). In that intertemporal context, Rawls' difference principle would result in a constant utility path, with no growth (Solow, 1974; Burmeister and Hammond, 1977). Rawls (1971) acknowledged that economic growth is necessary, because without adequate material resources a society cannot develop institutions that guarantee equal liberties to all. ${ }^{2}$ Wealth creation is necessary for the effective defense of rights and liberties. Welfarist considerations cannot be ignored.

The welfarist approach to development is different from the right-based approach. While the latter is deontological, the former is a consequentialist approach based on intertemporal welfare functions (i.e., criteria) ranking the distribution of welfare between present and future generations, and prescribing optimal growth paths. In this context, the emphasis is on the weight of the various generations in the objective function, with strong implications on discounting and intergenerational equity. These questions have been extensively addressed in the sustainability literature.

\subsection{Sustainability and minimal rights for future generations}

The tension between rights and welfare is particularly important when trying to define "sustainable development." In this respect, there is an important difference between weak and strong sustainability (Neumayer, 2013). Proponents of weak sustainability generally seek to sustain some notion of welfare, and define sustainability criteria to address the intergenerational equity issue. On the other hand, proponents of strong sustainability are concerned with the rights of future generations to inherit a good environmental quality, and argue for the sustainment of environmental assets. In practice, environmental issues are often addressed in setting quantitative targets (e.g., the cap in the Kyoto Protocol for climate change, or the habitat conservation objectives in the Nagoya Protocol for the Con-

\footnotetext{
${ }^{2}$ The need for adequate savings is a major concern for Rawls, because, "to establish effective, just institutions within which the basic liberties can be realized" society must have a sufficient material base. As a unmodified difference principle would lead to "no savings at all," he pointed out that the difference principle must be modified to allow for economic growth. For this purpose, he sketched a theory of "just saving" in which generations must "carry their fair share of the burden of realizing and preserving a just society." See Long (2007).
} 
vention on Biological Diversity), which can be interpreted as a way to set environmental rights for all generations.

The traditional criterion for evaluating intertemporal development paths is the discounted utility criterion. According to this criterion, a decrease in the utility level of a generation (no matter how disadvantaged this generation already is and how large is the considered sacrifice) can be justified by a sufficient increase in the utility level of some other generations. This criterion is strongly inequitable, and has been shown to display "dictatorship of the present" (Chichilnisky, 1996). For example, in the case of the Dasgupta-Heal-Solow model, the optimal consumption path under discounted utilitarianism decreases toward zero in the long run while the resource is exhausted (Dasgupta and Heal, 1974). Defining a criterion that accounts for intergenerational equity, and in particular for the long run, has been a challenge for economists addressing the sustainability issue (Heal, 1998; Martinet, 2012).

Another, extreme alternative is the Green Golden Rule (Chichilnisky et al., 1995), which defines the development path reaching and sustaining the highest possible development level. Giving weight only to the very long-run, this criterion implies "dictatorship of the future" (Chichilnisky, 1996). To avoid dictatorships of the present and the future, Chichilnisky (1996) suggested to use as a welfare function the weighted sum of the usual discounted stream of utilities and a measurement of the limiting behavior of the utility sequence. Alvarez-Cuadrado and Long (2009) proposed to modify Chichilnisky's criterion by replacing the second term with the minimal level of utility of the trajectory over time, in line with the maximin criterion (Solow, 1974; Cairns and Long, 2006). Considered alone, the maximin criterion is insensitive to the utilities of generations that are not the poorest. ${ }^{3}$ Whenever it is possible to smooth utility over time, the maximin principle leads to no growth, no matter how small is the initial maximal sustainable utility. There is no concern for growth, which may be an issue if capital accumulation is needed to develop and sustain just institutions. By considering a weighted average of the standard sum of discounted utilities and a Rawlsian part which places special emphasis on the utility of the least advantaged generation, the "Mixed Bentham-Rawls" (MBR) criterion provided by Alvarez-Cuadrado and Long (2009) satisfies both non-dictatorship of the present and of the future, just as Chichilnisky's welfare function. The maximization of the $M B R$ criterion determines endogenously a minimal utility level to be sustained forever. Without referring to rights, the criterion introduces the idea that the welfare of some generations

\footnotetext{
${ }^{3}$ The maximin criterion has been strengthened to eliminate some maximin paths that are Pareto dominated by other paths that have the same minimum level of utility. See Asheim and Zuber (2013); Long (2011).
} 
(in particular future generations) cannot be sacrificed too much for other generations (in particular present generations).

All the described intertemporal welfare functions weigh the utility of the various generations differently. This has strong implications in terms of discounting. More specifically, the discount rate to be used to evaluate project investment with long run impacts is strongly influenced by the criterion chosen. None, however, encompasses a concern for rights.

A challenge for strong sustainability and the practice of sustainable development is to define the quantities of environmental assets to be conserved or the levels of environmental indicators to be sustained. Martinet (2011) proposed a criterion which defines several minimal rights to be guaranteed to all generations. These rights are represented by a set of constraints on indicators, and the associated sustainability thresholds, which must be chosen optimally. This criterion does not permit intergenerational trade-offs. All generations have the same minimal rights. By not accounting for the utility levels of different generations in an intertemporal welfare function, the criterion solely defines minimal rights representing sustainability without considering welfare and the "cost" of satisfying these rights. This is an important limitation for the scope of application.

In the present paper, we aim at developing an approach which encompasses both welfarist considerations and the concern for rights. In particular, we emphasize that, if minimal rights are imposed to the development path, one should account for the consequences of these rights on welfare when setting their levels. We show that a criterion combining an index based on minimal rights with a welfare index can be used to define endogenously the levels of the minimal rights, accounting for the associated cost in terms of present-value welfare. This could represent the choice of a society defining (economic and environmental) minimal rights to be guaranteed over time to embody the idea of sustainability along a development path.

\section{The Rights and Welfare Indicator}

\subsection{Axiomatic foundation}

We start by characterizing a criterion satisfying a set of usual axioms along with some new axioms representing sensitivity to rights and to welfare. For this purpose, it is convenient to use a discrete time framework, as usual in the literature (see, e.g., Chichilnisky, 1996; Lauwers, 1997a,b, 1998; Alvarez-Cuadrado and Long, 2009).

We assume that the economy is composed of infinitely many generations, each liv- 
ing for one period, without overlapping. We make the simplifying assumption that each generation can be assimilated to a single, representative agent, and do not address intragenerational equity, in order to focus on the intertemporal dimension of the problem. Denoting the time index by $t=1,2, \ldots$, the utility of the generation $t$ is denoted by $u_{t}$, assumed to be bounded. We define a utility allocation as an infinite sequence $\mathbf{u} \equiv\left\{u_{t}\right\}_{t=1,2, \ldots} \equiv\left\{u_{1}, u_{2}, \ldots\right\}$. Let $S \subset \mathbb{R}^{\infty}$ be the set of all bounded utility sequences.

We also assume that society puts values on some rights to be guaranteed at all times. For example, a society may hold that all generations have a right to some basic consumption level, or some basic level of health care or education. From an environmentalist point of view, all generations may have the right to enjoy a pristine environment or a certain level of biodiversity. For each right, we suppose that it is possible to construct an ad hoc indicator measuring at each point of time how society is faring in terms of meeting that right. Consider a finite number $(I)$ of rights. The satisfaction of these rights can be assessed quantitatively by comparing the related indicators to thresholds $\mu_{i}$ (for $i=1, \ldots, I)$ with the convention that the indicators are defined such that higher scores represent better performances. This means that we formalize thresholds as floors rather than ceilings, normalizing so that indicators are "goods" rather than "bads." Formally, an indicator $\mathcal{I}$ is a function of a set of state variables $x$ and control variables $c$, resulting in constraints of the form $\mathcal{I}(x(t), c(t)) \geq \mu$ at all times $t$, where the $\mu$ are thresholds to be chosen optimally. The thresholds $\mu_{i}$ are then characterizing the levels at which the rights are set, and we assume that these minimal levels are the object of a social choice. Let $\boldsymbol{\mu}=\left(\mu_{1}, \ldots, \mu_{I}\right)$ denote a vector of finite thresholds within a bounded set of feasible thresholds $M \subset \mathbb{R}^{I}$ representing rights.

A social alternative is a couple $(\boldsymbol{\mu}, \boldsymbol{u}) \in M \times S$ corresponding to a vector of minimal rights $\boldsymbol{\mu}$ granted to all generations for deontological reasons, and an infinite utility sequence $\mathbf{u}$ which can be assessed by a welfare measure in a consequentialist perspective. A social ranking criterion is a preference order $\succsim$ on $M \times S$, i.e., a complete and transitive binary relation in $M \times S$ (Lauwers, 1997a,b). We aim at characterizing a real value function $\mathcal{P}: M \times S \rightarrow \mathbb{R}$ representing such a criterion.

We denote by $\ell_{\infty}$ the set of bounded infinite vectors. The ranking of social alternatives $(\boldsymbol{\mu}, \boldsymbol{u})$ is equivalent to the ranking of infinite vectors $\left(\mu_{1}, \ldots, \mu_{I}, u_{1}, u_{2}, \ldots, u_{t}, \ldots\right) \in \ell_{\infty}$, where the first $I$ elements are rights thresholds and the following elements an infinite sequence of utility. For the representability of such a preference order, we need to impose some conditions on the topology on $\ell_{\infty}$. We consider the strict or myopic topology $\mathcal{T}_{m}$ generated by the distance function $d_{m}(\mathbf{a}, \mathbf{b})=$ supremum $\left\{\left|a_{k}-b_{k}\right| / k \mid k \in \mathbb{N}_{0}\right\}$, with $(\mathbf{a}, \mathbf{b}) \in \ell_{\infty} \times \ell_{\infty}$. When $\ell_{\infty}$ is equipped with this topology, a continuous and monotonic 
preference order $\succsim$ upon $\ell_{\infty}$ is representable by a real, continuous map $\mathcal{P}^{I}: \ell_{\infty} \rightarrow \mathbb{R}$ (Lauwers, 1997b, Proposition 1). ${ }^{4}$ We require the social ranking criterion to satisfy the following monotonicity axioms on rights and welfare, as well as an axiom of continuity. When no equivalent is specified, properties applying on $(\boldsymbol{\mu}, \boldsymbol{u}) \in M \times S$ apply to $\left(\mu_{1}, \ldots, \mu_{I}, u_{1}, u_{2}, \ldots, u_{t}, \ldots\right) \in \ell_{\infty}$ ceteris paribus.

Axiom 1 (Monotonicity in right thresholds) $\left(\boldsymbol{\mu}^{\prime}, \boldsymbol{u}\right) \succsim(\boldsymbol{\mu}, \boldsymbol{u})$ if $\mu_{i}^{\prime} \geq \mu_{i}$ for all $i \in\{1, \ldots, I\}$.

Axiom 2 (Strong Pareto in welfare) $\left(\boldsymbol{\mu}, \boldsymbol{u}^{\prime}\right) \succ(\boldsymbol{\mu}, \boldsymbol{u})$ if $u_{t}^{\prime} \geq u_{t}$ for all $t$, with strict inequality for some $t$.

Lemma 1 (Monotonicity on $\ell_{\infty}$ ) A preference order $\succsim$ over $M \times S$ satisfying Monotonicity in right thresholds and Strong Pareto in welfare is equivalent to a monotonic preference order on $\ell_{\infty}$.

Axiom 3 (Continuity) The order $\succsim$ is continuous with respect to the strict topology.

The next two axioms characterize the relationship between rights and welfare within the evaluation of social alternatives. The axiom of Separability of Rights and Welfare specifies that the ranking of two social alternatives sharing the same vector of thresholds (respectively, the same utility sequence) is not reversed if the vector of thresholds (respectively, the utility sequence) is identically changed in both alternatives. The axiom of Linear Commensurability specifies that the value accorded to rights by the social criterion is commensurable with utility: it is as if there were a virtual agent that derives "utility" from the Rights thresholds.

Axiom 4 (Separability of Rights and Welfare) $(\boldsymbol{\mu}, \boldsymbol{u}) \succsim\left(\boldsymbol{\mu}^{\prime}, \boldsymbol{u}\right) \Leftrightarrow\left(\boldsymbol{\mu}, \boldsymbol{u}^{\prime}\right) \succsim$ $\left(\boldsymbol{\mu}^{\prime}, \boldsymbol{u}^{\prime}\right)$ and $(\boldsymbol{\mu}, \boldsymbol{u}) \succsim\left(\boldsymbol{\mu}, \boldsymbol{u}^{\prime}\right) \Leftrightarrow\left(\boldsymbol{\mu}^{\prime}, \boldsymbol{u}\right) \succsim\left(\boldsymbol{\mu}^{\prime}, \boldsymbol{u}^{\prime}\right)$

Separability of Rights and Welfare serves the same purpose as the usual separability axiom. A consequence of the Separability of Rights and Welfare axiom is that the preference function $\mathcal{P}^{I}$ can be written in the alternative form

$$
\mathcal{P}^{I}\left(\mu_{1}, \ldots, \mu_{I}, u_{1}, \ldots, u_{t}, \ldots\right)=\mathcal{P}^{0}\left(\mathcal{R}\left(\mu_{1}, \ldots, \mu_{I}\right), u_{1}, \ldots, u_{t}, \ldots\right)
$$

\footnotetext{
${ }^{4}$ In an infinite dimensional space, the continuity concept is sensitive to the choice of the distance function used. It may have some strong implications on the criterion. For example, the strict topology used here implies eventual impatience (Lauwers, 1997b).
} 
where $\mathcal{P}^{0}$ satisfies the same continuity and monotonicity properties as $\mathcal{P}^{I}$, and the right index $\mathcal{R}: M \rightarrow \mathbb{R}$ is continuous, non-decreasing in all its terms and assumed to be bounded. This is obtained by applying the same steps as the reformulation of welfare as an aggregator of immediate utility and prospective utility in Koopmans (1960, p.292-293). ${ }^{5}$

Axiom 5 (Linear Commensurability) The Right index $\mathcal{R}(\boldsymbol{\mu})$ is commensurable to utility, and the map $\mathcal{P}^{0}: \ell_{\infty} \rightarrow \mathbb{R}$ is linear, i.e., it satisfies $\mathcal{P}^{0}\left(\alpha_{1} \mathcal{R}\left(\boldsymbol{\mu}^{\mathbf{1}}\right)+\alpha_{2} \mathcal{R}\left(\boldsymbol{\mu}^{\mathbf{2}}\right), \alpha_{1} \boldsymbol{u}^{\mathbf{1}}+\right.$ $\left.\alpha_{2} \boldsymbol{u}^{\mathbf{2}}\right)=\alpha_{1} \mathcal{P}^{0}\left(\mathcal{R}\left(\boldsymbol{\mu}^{\mathbf{1}}\right), \boldsymbol{u}^{\mathbf{1}}\right)+\alpha_{2} \mathcal{P}^{0}\left(\mathcal{R}\left(\boldsymbol{\mu}^{\mathbf{2}}\right), \boldsymbol{u}^{\mathbf{2}}\right)$.

Linear Commensurability serves the same purpose as the independence axiom used in the literature. Under Linear Commensurability, the Right index $\mathcal{R}(\boldsymbol{\mu})$ is expressed in the same unit as utility, and can be interpreted as the utility $u_{0}$ of a virtual agent. Usual cardinal unit comparability applies, and the preferences over rights and welfare are representable by a continuous linear map (Chichilnisky, 1996; Lauwers, 1997b, 1998). According to the Yosida-Hewitt representation theorem (see Lauwers, 1998), a continuous linear map $G: \ell_{\infty} \rightarrow \mathbb{R}$ can be decomposed into a sum $G=G^{p}+G^{s}$ where $G^{p}$ is a purely finitely additive measure, i.e., $G^{p}(\mathbf{a})=0$ for all $\mathbf{a} \in \ell_{\infty}$ with at most a finite number of nonzero elements, and $G^{s}$ is $\ell_{1}$-summable, i.e., $G^{s}(\mathbf{a})=\sum_{k=1}^{\infty} \alpha_{k} a_{k}$ with $\mathbf{a} \in \ell_{\infty}$ and $\sum_{k=1}^{\infty} \alpha_{k}<\infty$.

The topology $\mathcal{T}_{m}$ is the largest topology such that the purely finitely additive measure in the decomposition is nil. A $\mathcal{T}_{m}$-continuous linear map is thus representable by a discounted sum exhibiting eventual impatience (Lauwers, 1997b). The preference function $\mathcal{P}^{0}$ can be written as $\mathcal{P}^{0}\left(\mathcal{R}\left(\mu_{1}, \ldots, \mu_{I}\right), u_{1}, \ldots, u_{t}, \ldots\right)=\alpha_{0} \mathcal{R}(\boldsymbol{\mu})+\sum_{t=1}^{\infty} \alpha_{t} u_{t}$, with $\sum_{t=0}^{\infty} \alpha_{t}<\infty$. The Right and Welfare Indicator can thus be characterized by the following theorem.

Theorem 1 (Right and Welfare Indicator) Under the axioms of Monotonicity in right thresholds, Strong Pareto in welfare, Continuity, Separability of Rights and Welfare and Linear Commensurability, a social ranking criterion over social alternatives defined by a vector of minimal rights $\boldsymbol{\mu}$ and an infinite sequence of utility $\boldsymbol{u}$ is characterized by a continuous and increasing real value function

$$
\mathcal{P}(\boldsymbol{\mu}, \boldsymbol{u})=\theta \mathcal{R}(\boldsymbol{\mu})+(1-\theta) \sum_{t=1}^{\infty} \beta_{t} u_{t}
$$

with $0 \leq \theta<1$ and $\sum_{t=1}^{\infty} \beta_{t}<\infty$.

\footnotetext{
${ }^{5}$ Here, the preference function is reformulated as a function of a right index and the utility sequence. We could even specify the function as an aggregator of a right index and welfare of the form $\tilde{\mathcal{P}}(\mathcal{R}(\boldsymbol{\mu}), \mathcal{W}(\boldsymbol{u}))$.
} 
The RWI is weakly sensitive both to rights and to welfare, without a preponderance of one on the other. ${ }^{6}$ In practical applications, we will focus on the case where $0<\theta<1$.

Eq. (1) clearly shows that a conflict may arise between respecting rights on the one hand and having a Pareto superior utility path on the other hand. For example, by insisting on some rights, such as the preservation of some virgin ecosystems forever for example, society may limit the utility of all generations. Giving up the associated right may improve the welfare of all generations, which would be efficient in a Pareto sense.

\subsection{RWI criteria in an economic modeling framework}

Theorem 1 characterizes a family of criteria combining rights and welfare as a weighted sum of a right and a welfare index. It, however, does not provide a unique functional form to maximize in the classical optimal control framework used in growth theory. In fact, optimal control requires strong conditions on differentiability which cannot easily be derived from axioms. We thus specify a particular criterion with additional differentiability properties, to be applied to usual economic modeling frameworks.

Economic modeling framework. We consider a stylized, general dynamic economic model. As most of the literature on optimal growth and sustainability criteria use continuous time, we adopt a continuous time framework hereafter. Time is denoted by $t$, with $t \in \mathbb{R}^{+}$. Let $x$ be a vector of $n$ state variables, and $c$ a vector of $m$ control variables. The initial state $x(0)=x_{0}$ is given. For the sake of simplicity, we consider time-autonomous problems. The dynamics equations are, for $k=1, \ldots, n$

$$
\dot{x}_{k}(t)=g_{k}(x(t), c(t))
$$

A time path is denoted by $(x(\cdot), c(\cdot))$. Following the standard control theoretic treatment, we require $x(\cdot)$ to be piece-wise differentiable and $c(\cdot)$ to be piece-wise continuous. For economic variables, stocks are typically restricted to be non-negative. Given the values of the state variables, the control variables at time $t$ must belong to a technologically

\footnotetext{
${ }^{6}$ One could define the concepts of preponderance of rights and preponderance of welfare as follows. Preponderance of rights: A social ranking criterion displays preponderance of rights if and only if $(\mathbf{u}, \boldsymbol{\mu}) \succ$ $\left(\mathbf{u}^{\prime}, \boldsymbol{\mu}^{\prime}\right)$ implies $\left(\mathbf{u}^{\#}, \boldsymbol{\mu}\right) \succ\left(\tilde{\mathbf{u}}, \boldsymbol{\mu}^{\prime}\right)$ for all vectors of minimal rights and utility sequences.

Preponderance of welfare: A social ranking criterion displays preponderance of welfare if and only if $(\mathbf{u}, \boldsymbol{\mu}) \succ\left(\mathbf{u}^{\prime}, \boldsymbol{\mu}^{\prime}\right)$ implies $\left(\mathbf{u}, \boldsymbol{\mu}^{\#}\right) \succ\left(\mathbf{u}^{\prime}, \tilde{\boldsymbol{\mu}}\right)$ for all vectors of minimal rights and utility sequences.

The RWI does not display preponderance of rights or preponderance of welfare. These properties are consequences of the monotonicity and continuity axioms in Theorem 1. In particular, continuity rules out lexicographic orders.
} 
feasibility set $A(x(t))$ which is characterized by $s$ inequality constraints:

$$
h_{j}(x(t), c(t)) \geq 0, \quad j=1,2, \ldots, s .
$$

Rights measurement. The indicators measuring the performance of the economy with respect to rights are functions of state and control variables $\mathcal{I}_{i}(x, c)$, with $i=1, \ldots, I$. An endogenously determined threshold for each indicator objectifies the level $\mu_{i}$ below which the indicator is not allowed to fall. For $i=1, \ldots, I$, and all times $t$, the constraints associated with minimal rights read

$$
\mathcal{I}_{i}(x(t), c(t)) \geq \mu_{i}
$$

The preferences of society over the minimal rights formalized by the thresholds are represented by a function $\mathcal{R}\left(\mu_{1}, \ldots, \mu_{I}\right)$, which is non-decreasing in all its arguments. This function can be interpreted as a right index. This index is an aggregate measure of the threshold levels, not of the extent to which the associated indicators exceed the various thresholds.

Welfare measurement. Instantaneous utility at time $t$ is derived from current state and control variables, and denoted by $U(x(t), c(t))$. Assuming a constant utility discount rate $\delta>0$, a feasible time path $(x(\cdot), c(\cdot))$ starting from state $x_{0}$ yields a welfare level

$$
\mathcal{W}(x(\cdot), c(\cdot)) \equiv \chi(\delta) \int_{0}^{\infty} e^{-\delta t} U(x(t), c(t)) d t
$$

where $\chi(\delta)$ is equal either to 1 or to $\delta$. We call these two alternative specifications the "absolute conception" and "relative conception" of discounting. Which conception is adopted has no effects on the comparison of welfare streams along alternative development paths; however, the choice between these two conceptions of discounting matters when doing comparative statics with respect to the discount rate within a mixed criterion (see our comparative static Result 7 in Subsection 5.2). ${ }^{7}$

Under the Commensurability axiom, the right index $\mathcal{R}$, i.e., the value granted to rights, is expressed in welfare equivalent units. The exact nature of this equivalent depends on how one conceptualizes discounting in the aggregation of welfare across generations. According to the "absolute conception" of discounting, i.e., when $\chi(\delta)=1$, welfare is

\footnotetext{
${ }^{7}$ In the literature on sustainability, Asheim and Ekeland (2016) adopted what we call the relative conception of discounting, while Alvarez-Cuadrado and Long (2009), Tol (2013), and Figuières et al. (2017) seemed to have in mind the absolute conception of discounting.
} 
measured in present utility terms, and only the welfare valuation of present utility is unaffected by a change of the discount rate. An increase in the discount rate $\delta$ has two effects: (a) for any arbitrary utility path it reduces the weights attached to utilities of more distant future generations while the weight attached to the current generation's utility stays constant at unity and (b) it reduces the social welfare of any constant utility path $u()=.\bar{u}$. Using the discrete time notations of our axiomatic characterization, under the absolute conception of discounting, welfare satisfies $\mathcal{W}\left(u_{1}, 0,0,0, \ldots\right)=u_{1}$, whereas $\mathcal{W}(\bar{u}, \bar{u}, \bar{u}, \bar{u}, \ldots)=\bar{u} / \delta$. In contrast, according to the "relative conception" of discounting, i.e., when $\chi(\delta)=\delta$, welfare is equal to the level of the equally distributed equivalent utility. Using the notations of the axiomatic characterization, welfare satisfies $\mathcal{W}(\bar{u}, \bar{u}, \bar{u}, \bar{u}, \ldots)=\bar{u}$, regardless of the discount rate. An increase in the discount rate $\delta$ (a) has no effect on the social welfare of any constant utility path $u()=.\bar{u}$, whereas (b) for any arbitrary utility path, it increases the weights given to utilities of near-term generations and reduces the weight given to the utilities of distant generations.

Combining rights and welfare in an indicator. The social problem of defining the optimal levels for minimal rights along with the optimal growth path can be represented as the maximization of a Rights and Welfare Indicator (RWI) defined as the weighted sum of the right index $\mathcal{R}(\boldsymbol{\mu})$ and the welfare index $\mathcal{W}(x(\cdot), c(\cdot))$, the relative weight given to "rights" being defined by a parameter $0<\theta<1$. The parameter $\theta$ is taken as given and can be interpreted as the political weight of the "non-welfarist" proponents.

The resulting optimal control problem with endogenous constraints is defined as follows.

$$
\begin{array}{ll}
\max _{\boldsymbol{\mu}, c(\cdot)} & \theta \mathcal{R}\left(\mu_{1}, \ldots, \mu_{I}\right)+(1-\theta) \mathcal{W}(x(\cdot), c(\cdot)) \\
\text { s.t. } & \begin{array}{ll}
x(0)=x_{0} \text { and } \forall t: \\
\dot{x}_{k}(t)=g_{k}(x(t), c(t)), & k=1, \ldots, n, \\
& h_{j}(x(t), c(t)) \geq 0, \quad j=1, \ldots, s, \\
\mathcal{I}_{i}(x(t), c(t)) \geq \mu_{i}, \quad i=1, \ldots, I .
\end{array}
\end{array}
$$

This problem defines jointly the optimal growth path and the optimally chosen levels of the rights. Increasing the threshold for any right may involve a cost in terms of welfare that the optimization must account for. The next section characterizes the resulting trade-offs. 


\section{Optimal choice of minimal rights and growth path}

In this section, we first provide general results on the trade-offs between rights and welfare. We then provide a direct approach to the maximization of the RWI, defining jointly the minimal rights and the development path.

\subsection{Characterization of the trade-offs between Rights and Wel- fare}

The optimization problem (6) can be formulated in a more intuitive way by introducing the following notations.

For any vector of thresholds $\left(\mu_{1}, \ldots, \mu_{I}\right)$, define $\mathcal{F}\left(x_{0} ; \mu_{1}, \ldots, \mu_{I}\right)$ as the set of all the economic paths $(x(\cdot), c(\cdot))$ starting from initial state $x_{0}$ and satisfying constraints $(2,3,4)$ :

$$
\mathcal{F}\left(x_{0} ; \mu_{1}, \ldots, \mu_{I}\right)=\left\{\begin{array}{l|l}
(x(\cdot), c(\cdot)) & \begin{array}{ll}
x(0)=x_{0} \text { and } \forall t: \\
\dot{x}_{k}(t)=g_{k}(x(t), c(t)), & k=1, \ldots, n \\
h_{j}(x(t), c(t)) \geq 0, & j=1, \ldots, s \\
\mathcal{I}_{i}(x(t), c(t)) \geq \mu_{i}, & i=1, \ldots, I
\end{array}
\end{array}\right\}
$$

Clearly, given the initial stock $x_{0}$, the set $\mathcal{F}\left(x_{0} ; \mu_{1}, \ldots, \mu_{I}\right)$ may be empty if thresholds $\boldsymbol{\mu}$ are too high. It is sensible to consider only thresholds that are consistent with the endowment $x_{0}$. Since the satisfaction of minimal rights, i.e., the maintenance of an indicator above a threshold level, typically requires the use of resources, it is plausible to argue that for any given level of resource endowment, there is a well-defined set of feasible thresholds within which a vector of optimal thresholds would be chosen.

Definition 1 (Set of feasible thresholds) Given an initial state $x_{0}$, the set of feasible thresholds is defined as the set of thresholds for which there are feasible economic paths starting from state $x_{0}$ and satisfying constraints (4) at all times, i.e.,

$$
\mathcal{M}\left(x_{0}\right)=\left\{\left(\mu_{1}, \ldots, \mu_{I}\right) \mid \mathcal{F}\left(x_{0} ; \mu_{1}, \ldots, \mu_{I}\right) \neq \emptyset\right\}
$$

We assume that the set of feasible thresholds $\mathcal{M}\left(x_{0}\right)$ is delimited by a threshold possibility frontier, represented by a differentiable function $\phi$. This upper boundary is represented by the equality $\phi\left(\mu_{1}, \ldots, \mu_{I} ; x_{0}\right)=0$, where $\phi$ is a real-valued differentiable function, with the convention that points below this frontier yield $\phi\left(\mu_{1}, \ldots, \mu_{I} ; x_{0}\right)>0$. Given $x_{0}$, any vector of thresholds $\mu_{1}, \ldots, \mu_{I}$ that lies beyond the frontier would yield $\phi\left(\mu_{1}, \ldots, \mu_{I} ; x_{0}\right)<0$. Then, for any feasible vector of thresholds, the non-negative, real 
number $\phi\left(\mu_{1}, \ldots, \mu_{I} ; x_{0}\right)$ can be thought of as a measure of how much leeway there is left for satisfying objectives other than the chosen minimal rights. Since higher thresholds reduce the leeway, we suppose that $\partial \phi / \partial \mu_{i} \leq 0$. On the boundary of the set of feasible thresholds, one cannot increase all the minimal rights together, reflecting conflicts among minimal rights. In the case of two thresholds, this trade-off is characterized by the negative slope of the threshold possibility frontier $\frac{d \mu_{2}}{d \mu_{1}}=-\frac{\phi_{\mu_{1}}}{\phi_{\mu_{2}}} \leq 0$.

It is likely that increasing any threshold will reduce the welfare index. In this sense, there is a tension between rights and welfare. To emphasize this, we define the constrained welfare value function as the maximal welfare level which can be achieved given some rights constraints.

Definition 2 (Constrained welfare value function) For any vector of feasible thresholds $\left(\mu_{1}, \ldots, \mu_{I}\right) \in \mathcal{M}\left(x_{0}\right)$, we define the associated constrained welfare value function $V\left(x_{0} ; \mu_{1}, \ldots, \mu_{I}\right)$ as

$$
\begin{aligned}
V\left(x_{0} ; \mu_{1}, \ldots, \mu_{I}\right) \equiv \max _{(x(\cdot), c(\cdot))} \mathcal{W}(x(\cdot), c(\cdot)) \\
\text { s.t. } \quad(x(\cdot), c(\cdot)) \in \mathcal{F}\left(x_{0} ; \mu_{1}, \ldots, \mu_{I}\right)
\end{aligned}
$$

The following proposition states that increasing one or several minimal rights thresholds reduces the potential welfare. ${ }^{8}$

Proposition 1 (Tension between rights and welfare) For any pair of threshold vectors $\left(\mu_{1}, \ldots, \mu_{I}\right)$ and $\left(\mu_{1}^{\prime}, \ldots, \mu_{I}^{\prime}\right)$ such that $\mu_{i}^{\prime} \geq \mu_{i}$ for $i=1, \ldots, I$, one has $V\left(x_{0} ; \mu_{1}^{\prime}, \ldots, \mu_{I}^{\prime}\right) \leq V\left(x_{0} ; \mu_{1}, \ldots, \mu_{I}\right)$.

Society may choose thresholds inside the feasibility set $\mathcal{M}\left(x_{0}\right)$, because the cost of being on the frontier, measured in terms of forgone consumption of some goods and services, may outweigh the value of guaranteeing a high level of rights. An optimal threshold vector should precisely balance the "costs" of thresholds in terms of welfare and the "moral worth" of thresholds in terms of rights.

Given previous definitions, the dynamic optimization problem (6) reduces to the following static optimization problem on rights thresholds, based on the constrained welfare value function $V\left(x_{0} ; \mu_{1}, \ldots, \mu_{I}\right)$ and on the frontier $\phi\left(\mu_{1}, \ldots, \mu_{I} ; x_{0}\right)$ of the set of feasible thresholds.

\footnotetext{
${ }^{8}$ Proofs are in the Appendix.
} 


$$
\begin{array}{ll}
\max _{\boldsymbol{\mu}} & \theta \mathcal{R}\left(\mu_{1}, \ldots, \mu_{I}\right)+(1-\theta) V\left(x_{0} ; \mu_{1}, \ldots, \mu_{I}\right) \\
\text { s.t. } & \phi\left(\mu_{1}, \ldots, \mu_{I} ; x_{0}\right) \geq 0 .
\end{array}
$$

This optimization problem can be solved using the following Lagrangian:

$$
\mathcal{L}=\theta \mathcal{R}\left(\mu_{1}, \ldots, \mu_{I}\right)+(1-\theta) V\left(x_{0} ; \mu_{1}, \ldots, \mu_{I}\right)+\gamma \phi\left(\mu_{1}, \ldots, \mu_{I} ; x_{0}\right),
$$

where $\gamma$ is the multiplier associated with the admissibility constraint for thresholds, which satisfies $\gamma \geq 0, \phi\left(\mu_{1}, \ldots, \mu_{I} ; x_{0}\right) \geq 0$, and $\gamma \phi()=$.0 . Optimality of the threshold levels requires the following first order conditions to be fulfilled for $i=1, \ldots, I$ :

$$
\theta \mathcal{R}_{\mu_{i}}^{\prime}+(1-\theta) \frac{\partial V}{\partial \mu_{i}}+\gamma \phi_{\mu_{i}}^{\prime}=0
$$

The first term of condition (10) is the weighted marginal contribution of the right to the Right index. The second term is the weighted marginal effect of the right on welfare. The non-positive term $\gamma \phi_{\mu_{i}}^{\prime}$ measures the cost of increasing the threshold $\mu_{i}$ marginally along the threshold possibility frontier, as this increase necessitates a decrease in a the threshold of some other right.

From these conditions, distinguishing interior and corner solutions, we can derive the following results characterizing the tension between rights and welfare.

Consider first any interior solution of problem (8), with $\phi\left(\mu_{1}^{\star}, \ldots, \mu_{I}^{\star} ; x_{0}\right)>0$ (i.e., the optimal thresholds are not on the boundary of the set of feasible thresholds) and thus $\gamma=0$. Eq. (10) then reduces to $\theta \mathcal{R}_{\mu_{i}}^{\prime}+(1-\theta) \frac{\partial V}{\partial \mu_{i}}=0$ for all $i$. All the rights are set according to their respective welfare cost, which can be interpreted as the social willingness-to-pay for the setting of some moral or ethical norms. This illustrates the trade-offs between rights and welfare.

Result 1 (Welfare-based rights setting) In the absence of conflicts among minimal rights, at an interior solution the level of each right is set so that its marginal contribution to the RWI is nil. Its positive, weighted marginal contribution to the right index balances its negative, weighted marginal effect on welfare.

Consider now a boundary solution of problem $(8)$, such that $\phi(\cdot)=0$ (i.e., the optimal thresholds are on the boundary of the set of feasible thresholds) and thus $\gamma \geq 0$. Eq. (10) can be expressed as $\theta \mathcal{R}_{\mu_{i}}^{\prime}+(1-\theta) \frac{\partial V}{\partial \mu_{i}}=-\gamma \phi_{\mu_{i}}^{\prime} \geq 0$, which implies $\theta \mathcal{R}_{\mu_{i}}^{\prime} \geq-(1-\theta) \frac{\partial V}{\partial \mu_{i}}$ for 
all $i$ as $\phi_{\mu_{i}}^{\prime} \leq 0$ and $\frac{\partial V}{\partial \mu_{i}} \leq 0$. In this type of non-interior solution, rights conflict with one another in the sense that it is not possible to increase a minimal right without decreasing another one. Society would be ready to bear additional welfare costs to increase some minimal rights but it would require a reduction in some other rights. This illustrates the trade-offs among minimal rights. In this case, where the optimal right thresholds are on the threshold possibility frontier, the optimal choice of rights as well as the optimal development path are largely driven by trade-offs among minimal rights (even though the welfare costs of rights are also taken into account). ${ }^{9}$

Result 2 (Right-based solution) When there are conflicts among minimal rights, the level of some minimal rights may be limited in spite of a positive net marginal contribution to the RWI (in the sense that its positive, weighted marginal contribution to the right index is larger than its negative, weighted marginal effect on welfare).

Last, consider an interior solution of problem (8), where $\frac{\partial V}{\partial \mu_{i}}=0$ for some $i$. In this case, the corresponding minimal right is not constraining welfare maximization, and its level can be increased up to the point at which its marginal contribution to the right index is nil, $\mathcal{R}_{\mu_{i}}^{\prime}=0$. Otherwise, the minimal right is set at a lower level, in spite of its positive marginal value to the right index alone, to account for the welfare cost of the rights (as described in Result 1).

Result 3 (No tensions between a right and welfare) A particular right $\mu_{i}$ is set so as to maximize the right index $\left(\mathcal{R}_{\mu_{i}}^{\prime}=0\right)$ if and only if this right does not conflict with other rights (interior solution with $\gamma=0$ ) and has no welfare cost, i.e., $\frac{\partial V}{\partial \mu_{i}}=0$.

Although this analysis allows us to exhibit the tension between rights and welfare, it requires knowing the set of admissible thresholds $\mathcal{M}\left(x_{0}\right)$ and the constrained welfare value function $V\left(x_{0}, \boldsymbol{\mu}\right)$. Unfortunately, a full characterization of the set of feasible thresholds is a problem in and of itself (Martinet, 2011), and for most optimization problems, determining the value function is a difficult task (Leonard and Long, 1991). We thus develop a direct approach to solve problem (6) and get further results on the tension between rights and welfare.

\subsection{A direct approach to the maximization of the RWI}

In this section, we propose a direct approach to maximize the RWI. Since the right thresholds are constants to be chosen optimally, the optimization problem (6) is an optimal

\footnotetext{
${ }^{9}$ Contrary to Martinet (2011), here rights are not set to maximize the preferences defined over right thresholds, rather they are chosen to maximize the RWI, which gives a positive weight to welfare.
} 
control problem with endogenous constraints in which $\left(\mu_{1}, \ldots, \mu_{I}\right)$ are treated as control parameters. Maximizing the RWI is equivalent to solving the following optimization problem by choosing optimal $\boldsymbol{\mu}$ and $c(\cdot)$, given the initial state, the dynamics, and the admissibility and right constraints:

$$
\begin{aligned}
\max _{\boldsymbol{\mu}, c(\cdot)} & \int_{0}^{\infty}\left\{\delta \theta \mathcal{R}\left(\mu_{1}, \ldots, \mu_{I}\right)+(1-\theta) \chi(\delta) U(x(t), c(t))\right\} e^{-\delta t} d t . \\
\text { s.t. } & x(0)=x_{0}, \text { dynamics }(2) \text { and constraints (3) and }(4) .
\end{aligned}
$$

Let the vector of co-state variables be denoted by $\pi(t)$, the vector of multipliers associated with the inequality constraints (3) by $\lambda(t)$, and the vector of multipliers associated with the right-based constraints (4) by $\omega(t)$. The current values of these variables are denoted respectively by $\psi(t)=e^{\delta t} \pi(t), \Delta(t)=e^{\delta t} \lambda(t)$ and $w(t)=e^{\delta t} \omega(t)$. The current-value Hamiltonian of problem (11) is

$$
H^{c}=\delta \theta \mathcal{R}\left(\mu_{1}, \ldots, \mu_{I}\right)+(1-\theta) \chi(\delta) U(x, c)+\psi g(x, c)
$$

and the current-value Lagrangian is

$$
L^{c}=H^{c}+\Delta h(x, c)+w[\mathcal{I}(x, c)-\mu]
$$

The necessary conditions for such problems can be derived from Hestenes' Theorem. ${ }^{10}$ From the necessary conditions and the usual transversality conditions stated in the Appendix, we can further characterize the nature of the welfare cost of rights. For technical reasons, we need to distinguish the case of rights defined as pure state constraints ${ }^{11}$ from the general form (4). Some minimal rights may correspond to a requirement to keep a particular capital stock above a threshold, corresponding to a level to be transmitted to future generations. It could be the case for natural resources, in a strong sustainability perspective. We refer to these rights as legacy constraints, whereas the general form of rights is called needs satisfaction.

Legacy constraints: We assume that, among the $I$ minimal rights, the $p$ firsts are

\footnotetext{
${ }^{10}$ See Leonard and Long (1991, Theorem 7.11.1) or Takayama (1985) for an exposition of Hestenes' Theorem which deals with optimal control problems involving control parameters and various constraints. The detail of these necessary conditions, as well as the proofs of the following propositions, are presented in the Appendix.

${ }^{11}$ In this case, the induced constraint can be binding in the limiting sense, and one needs to specify a particular 'terminal manifold' for the associated constraint, as well as a specific terminal condition. The transversality conditions with respect to these stocks are $\lim _{t \rightarrow \infty} \pi_{i}(t) \geq 0$ and $\lim _{t \rightarrow \infty} \pi_{i}(t)\left(x_{i}(t)-\mu_{i}\right)=$ 0 .
} 
legacy constraints. Without loss of generality, we suppose that a legacy constraint applies to each of the $p$ firsts state variables, where $0 \leq p \leq n$. The indicator associated to each legacy minimal rights is simply the corresponding state variable: $\mathcal{I}_{i}(x, c) \equiv x_{i}$ for $i=1,2, \ldots, p$.

Needs satisfaction rights: The general type of rights corresponds to the satisfaction of some needs at all times. The associated indicators depend on decision variables (and possibly on state variables too) and are of the form $\mathcal{I}_{i}(x, c)$ for $i=p+1, \ldots, I$.

The constraints associated to both types of rights read

$$
\begin{array}{rlrl}
x_{i}(t) & \geq \mu_{i}, & i=1, \ldots, p, & \forall t . \\
\mathcal{I}_{i}(x(t), c(t)) & \geq \mu_{i}, & i=p+1, \ldots, I, \quad \forall t .
\end{array}
$$

From the necessary conditions with respect to the optimal choice of the control parameters $\mu_{i}$ (eqs. (30) and (31) in the appendix), the rights thresholds must satisfy the following conditions, derived from eqs. (32) and (33) in the appendix:

$$
\theta \mathcal{R}_{\mu_{i}}^{\prime}-\int_{0}^{\infty} e^{-\delta t} w_{i}(t) d t= \begin{cases}\bar{\pi}_{i} & i=1, \ldots, p \\ 0 & i=p+1, \ldots, I\end{cases}
$$

with $\bar{\pi}_{i} \equiv \lim _{t \rightarrow \infty} \pi_{i}(t)$.

Combining this equation with eq. (10), we obtain the following result characterizing the opportunity cost of rights.

Result 4 (Opportunity cost of a right) The marginal opportunity cost of a right threshold $\mu_{i}^{\star}$ in terms of welfare and conflicts with other rights is equal to the discounted sum of the shadow cost of the associated constraint at all times (including the terminal cost for legacy rights), i.e.,

$$
(1-\theta) \frac{\partial V}{\partial \mu_{i}}+\gamma \phi_{\mu_{i}}^{\prime}= \begin{cases}-\int_{0}^{\infty} e^{-\delta t} w_{i} d t-\bar{\pi}_{i} & i=1, \ldots, p \\ -\int_{0}^{\infty} e^{-\delta t} w_{i} d t & i=p+1, \ldots, I\end{cases}
$$

The opportunity cost of a right $\mu_{i}^{\star}$ thus depends on whether or not (and when) the associated constraint is binding, including in the limiting sense for legacy constraints.

Result 4 allows us to specify the conditions under which Results 1, 2 and 3 apply.

Result 5 (Binding rights constraints) When a minimal right has a strictly positive marginal contribution to the right index at the optimum, i.e., $\mathcal{R}_{\mu_{i}}^{\prime}\left(\mu^{\star}\right)>0$, the associated constraint must be binding along the optimal development path, in the sense that there 
is a time interval $\left[t_{i}, t_{i}+\varepsilon_{i}\right]$ for some $\varepsilon_{i}>0$ such that $\mathcal{I}_{i}\left(x^{\star}(t), c^{\star}(t)\right)=\mu_{i}^{\star}$ for all $t \in$ $\left[t_{i}, t_{i}+\varepsilon_{i}\right]$. The constraint can be binding in the limiting sense for legacy rights.

Result 6 (No tension between rights and welfare) A minimal right has a nil marginal contribution to the right index at the optimum, i.e., $\mathcal{R}_{\mu_{i}}^{\prime}\left(\mu^{\star}\right)=0$, if and only if the associated constraint is never binding. ${ }^{12}$

We can interpret these results as follows: A minimal right induces an opportunity cost, either in terms of welfare loss or in terms of conflicts with other minimal rights, only if the associated constraint is binding (including in the limiting sense for legacy constraints).

\section{An example: The production-consumption econ- omy with a nonrenewable resource}

We apply the criterion to a canonical model often used in the sustainability literature: the Dasgupta-Heal-Solow model of nonrenewable resource extraction and capital accumulation (Dasgupta and Heal, 1974, 1979; Solow, 1974).

\subsection{The model}

The capital stock is denoted by $K(t)$, the resource stock by $S(t)$, resource extraction by $r(t) \geq 0$ and consumption by $c(t) \geq 0$, all defined as non-negative variables. We assume a Cobb-Douglas production function, i.e., $F(K, r)=K^{\alpha} r^{\beta}$, with $0<\beta<\alpha<1$ and $\alpha+\beta \leq 1$. The dynamics of this economy are as follows:

$$
\begin{aligned}
\dot{K}(t) & =K(t)^{\alpha} r(t)^{\beta}-c(t), \\
\dot{S}(t) & =-r(t),
\end{aligned}
$$

subject to the initial conditions $K(0)=K_{0}, S(0)=S_{0}$, and the terminal constraints,

$$
\lim _{t \rightarrow \infty} K(t) \geq 0 \text { and } \lim _{t \rightarrow \infty} S(t) \geq 0
$$

Instantaneous utility is derived only from consumption, i.e., $U(c(t))$, with $U^{\prime}(c)>0$, $\lim _{c \rightarrow 0} U^{\prime}(c)=+\infty$ and $U^{\prime \prime}(c)<0$. Several authors used this simple productionconsumption economy to address the climate change issue (e.g., Stollery, 1998; D'Autume

\footnotetext{
${ }^{12}$ In the case of legacy rights associated to pure-state-variable constraint, 'never binding' means that both $w_{i}(t)=0$ for all $t$ and $\bar{\pi}_{i}=0$.
} 
et al., 2010). The nonrenewable resource is related to fossil energy. Stabilizing green house gas (GHG) concentrations requires limiting the cumulative emissions over time. The inground resource stock is used as a proxy for non-emitted GHG. A limit on cumulative emissions can be represented by a constraint on resource extraction: a part of the stock has to be preserved forever. We thus consider two sustainability indicators : $\mathcal{I}_{1}(c, r, S, K) \equiv c$ and $\mathcal{I}_{2}(c, r, S, K) \equiv S$, so that the program is subject to the following minimal rights (sustainability constraints):

$$
\begin{aligned}
c(t) & \geq \mu_{c} \geq 0 \\
S(t) & \geq \mu_{S} \geq 0 .
\end{aligned}
$$

These constraints state that every generation has the right to a minimal consumption at level $\mu_{c}$, i.e., to a minimal utility level, and the right to a minimal preserved resource stock $\mu_{S}$.

Assume that $\mathcal{R}\left(\mu_{c}, \mu_{S}\right) \equiv \eta_{c} \mu_{c}+\eta_{S} \mu_{S}$, where $\eta_{c}$ and $\eta_{S}$ are strictly positive parameters, and consider the objective function:

$$
J\left(\mu_{c}, \mu_{S}, c(\cdot)\right) \equiv \theta\left[\eta_{c} \mu_{c}+\eta_{s} \mu_{S}\right]+(1-\theta) \chi(\delta) \int_{0}^{\infty} e^{-\delta t} U(c(t)) d t,
$$

to be maximized subject to dynamics (18) and (19) and constraints (21), (22), given the initial state $\left(K_{0}, S_{0}\right)$. Note that the satisfaction of constraint (22) at initial time implies $\mu_{S} \leq S_{0}$

\subsection{The indirect approach}

The set of achievable minimal consumption $\mu_{c}$ and preserved resource stock $\mu_{S}$ can be characterized by the restrictions $\mu_{c} \geq 0,0 \leq \mu_{S} \leq S_{0}$, together with the following relationship (see Martinet and Doyen, 2007; Martinet, 2011):

$$
\phi\left(\mu_{c}, \mu_{S}, K_{0}, S_{0}\right) \equiv(1-\beta)\left(\left(S_{0}-\mu_{S}\right)(\alpha-\beta)\right)^{\frac{\beta}{1-\beta}} K_{0}^{\frac{\alpha-\beta}{1-\beta}}-\mu_{c} \geq 0 .
$$

The upper boundary of this set satisfies $\phi\left(\mu_{c}, \mu_{S}, K_{0}, S_{0}\right)=0$ and can be represented by the threshold possibility frontier $\bar{\mu}_{c}\left(\mu_{S}\right)=(1-\beta)\left(\left(S_{0}-\mu_{S}\right)(\alpha-\beta)\right)^{\frac{\beta}{1-\beta}} K_{0}^{\frac{\alpha-\beta}{1-\beta}}$, defined for $\mu_{S} \in\left[0, S_{0}\right]$. This curve has a negative slope and is concave for all $\mu_{S}<S_{0}$, as $\frac{\partial \mu_{c}}{\partial \mu_{S}}=-\beta\left(S_{0}-\mu_{S}\right)^{\frac{-1}{1-\beta}}(\alpha-\beta)^{\frac{\beta}{1-\beta}} K_{0}^{\frac{\alpha-\beta}{1-\beta}}<0$, and $\frac{\partial^{2} \mu_{c}}{\left(\partial \mu_{S}\right)^{2}}=-\frac{\beta}{1-\beta}\left(S_{0}-\mu_{S}\right)^{\frac{-2+\beta}{1-\beta}}(\alpha-$ $\beta)^{\frac{\beta}{1-\beta}} K_{0}^{\frac{\alpha-\beta}{1-\beta}}<0$. 
The optimal threshold point $\left(\mu_{c}^{\star}, \mu_{S}^{\star}\right)$ may lie on the threshold possibility frontier or below it. If the relative weight $\theta /(1-\theta)$ is sufficiently large, i.e., if the weight accorded to minimal rights is relatively high with respect to that of welfare in the RWI indicator, $\left(\mu_{c}^{\star}, \mu_{S}^{\star}\right)$ will lie on the threshold possibility frontier. To see this, consider the constrained welfare value function $V\left(S_{0}-\mu_{S}, K_{0}, \mu_{c}\right)$ associated with the thresholds $\mu_{S}$ and $\mu_{c}$ :

$$
\begin{aligned}
V\left(S_{0}-\mu_{S}, K_{0}, \mu_{c}\right)= & \max _{c(\cdot), r(\cdot)} \chi(\delta) \int_{0}^{\infty} U(c(t)) e^{-\delta t} d t \\
\text { s.t. } \quad & K(0)=K_{0}, \quad S(0)=S_{0}-\mu_{S},
\end{aligned}
$$

dynamics $(18,19)$ and constraints $(21)$ and $(22)$.

The function $V(., .,$.$) is decreasing in \mu_{S}$ and in $\mu_{c}$. On the other hand, $\mathcal{R}(.,$.$) is$ strictly increasing and linear in $\mu_{S}$ and $\mu_{c}$. Since the RWI assigns a weight of $\theta$ to the right indicator, it follows that if $\theta$ is sufficiently close to unity, the maximization of $R W I$ with respect to $\mu_{c}$ and $\mu_{S}$ will give an optimal minimal rights vector on the threshold possibility frontier (Martinet, 2011). We conclude that if $\theta /(1-\theta)$ is large enough, the optimal thresholds point $\left(\mu_{c}^{\star}, \mu_{S}^{\star}\right)$ is on the threshold possibilities frontier. Conversely, if $\theta /(1-\theta)$ is not too high, the optimal thresholds point $\left(\mu_{c}^{\star}, \mu_{S}^{\star}\right)$ is in the interior of the threshold possibility frontier. When $\theta$ is sufficiently low (at least in the limiting case where $\theta$ tends toward zero), one gets the usual unconstrained discounted utility solution and $\left(\mu_{c}^{\star}, \mu_{S}^{\star}\right)$ is at the origin $(0,0)$. Depending on the relative importance of Rights and Welfare in the RWI indicator, the optimal solution is thus either on the threshold possibility frontier or within the set of feasible thresholds, allowing us to distinguish two types of development paths: Right-based sustainable development paths, and Constrained utilitarian paths.

Right-based sustainable development path. When the optimal thresholds $\left(\mu_{c}^{\star}, \mu_{S}^{\star}\right)$ are on the threshold possibility frontier, the optimal consumption is constant. ${ }^{13,14}$ The

\footnotetext{
${ }^{13}$ Suppose, on the contrary, that $c(t) \geq \mu_{c}^{\star}+\varepsilon$ over some time interval $\left[t_{1}, t_{2}\right]$ where $\varepsilon>0$. Then society could choose instead to consume only $\mu_{c}^{\star}+\kappa \varepsilon$ for some very small $\kappa$, where $0<\kappa<1$, and use the amount $(1-\kappa) \varepsilon$ of output thus saved to invest in the physical capital. This addtional investment would allow greater output in all future periods $t \geq t_{1}$, permitting consumption $c(t)$ to exceed $\mu_{c}^{\star}$ for all $t \geq t_{1}$. Furthermore, consumption in the time interval $\left[0, t_{1}\right]$ can also be increased above $\mu_{c}^{*}$ by extracting more resources. (Less resources would then be available after $t_{2}$, but this can be more than compensated for thanks to the higher $K\left(t_{2}\right)$ that results from the additional savings over the time interval $\left[t_{1}, t_{2}\right]$.) This means that the point $\left(\mu_{c}^{\star}+\kappa \varepsilon, \mu_{S}^{\star}\right)$ belongs to the feasible set $\mathcal{M}\left(x_{0}\right)$, contradicting the hypothesis that the solution $\left(\mu_{c}^{\star}, \mu_{S}^{\star}\right)$ is on the threshold possibility frontier.

${ }^{14}$ It can be shown that this constant consumption path maximizes the constrained welfare function, and that it can also satisfy the optimality conditions of the direct approach to the RWI maximization if the weight given to welfare $(1-\theta)$ is sufficiently small relative to the weight given to the minimal
} 
solution corresponds to the maximin consumption under a resource preservation constraint (Solow, 1974; Cairns and Long, 2006; Martinet and Doyen, 2007; Martinet, 2011). The consumption is constant, at a level

$$
c^{+}\left(K_{0}, S_{0}, \mu_{S}^{\star}\right)=(1-\beta)\left(\left(S_{0}-\mu_{S}^{\star}\right)(\alpha-\beta)\right)^{\frac{\beta}{1-\beta}} K_{0}^{\frac{\alpha-\beta}{1-\beta}}=\mu_{c}^{\star} .
$$

It yields a welfare $\mathcal{W}=\frac{\chi(\delta)}{\delta} U\left(\mu_{c}^{\star}\right)$ and the constraints yield a right index $\mathcal{R}\left(\mu_{c}^{\star}, \mu_{S}^{\star}\right)$, so that the maximized RWI level is $J=\theta \mathcal{R}\left(\mu_{c}^{\star}, \mu_{S}^{\star}\right)+(1-\theta) \frac{\chi(\delta)}{\delta} U\left(\mu_{c}^{\star}\right)$.

We know $\mu_{S}^{\star}$ as a function of $\mu_{c}^{\star}$ when these parameters are on the boundary of the feasibility set from the expression $\phi=0$. We can define the function $\mu_{S}^{\star}=\bar{\mu}_{S}\left(\mu_{c}^{\star}\right)$ from eq. (26). From the expression of $J$, and the first order condition on the optimal choice of the threshold parameters $\left(\frac{d J}{d \mu_{c}}=0\right)$, we can characterize the optimal rights: ${ }^{15}$

$$
\underbrace{(1-\theta) \frac{\chi(\delta)}{\delta} U^{\prime}\left(\mu_{c}^{\star}\right)}_{\begin{array}{c}
\text { Net present value gain } \\
\text { from increasing the } \\
\text { constant consumption level }
\end{array}}+\underbrace{\theta \mathcal{R}_{\mu_{c}}^{\prime}\left(\mu_{c}^{\star}, \bar{\mu}_{S}\left(\mu_{c}^{\star}\right)\right)}_{\begin{array}{c}
\text { Gain in terms of } \\
\text { guaranteed consumption }
\end{array}}=\underbrace{\theta \bar{\mu}_{S}^{\prime}\left(\mu_{c}^{\star}\right) \mathcal{R}_{\mu_{S}}^{\prime}\left(\mu_{c}^{\star}, \bar{\mu}_{S}\left(\mu_{c}^{\star}\right)\right)}_{\text {Loss in terms of preserved stock }}
$$

This equation can be re-arranged as follows

$$
\frac{(1-\theta) \chi(\delta) \delta^{-1} U^{\prime}\left(\mu_{c}^{\star}\right)+\theta \eta_{c}}{\theta \eta_{S}}=-\bar{\mu}_{S}^{\prime}\left(\mu_{c}^{\star}\right)
$$

It has a familiar interpretation: The left-hand side is the marginal rate of substitution of $\mu_{c}$ for $\mu_{S}$ along a RWI indifference curve, and the right-hand side is the marginal rate of transformation of the consumption right threshold into the resource-legacy right threshold along the threshold possibility frontier. From this equation, we can obtain the following comparative statics results: how do small changes in the preferences parameters $\delta, \theta, \eta_{c}$ and $\eta_{S}$ affect the optimal threshold $\mu_{c}$, assuming that the changes are small enough so that the solution pair $\left(\mu_{c}^{\star}, \mu_{S}^{\star}\right)$ remains on the threshold possibility frontier.

Result 7 (Effect of the discount rate) For a right-based development path, the marginal effect of the discount rate on the optimal minimal rights depends on the welfare definition.

\footnotetext{
consumption right $\theta \eta_{c}$. The mathematical details are available upon request.

${ }^{15}$ Providing an explicit expression of the optimal thresholds is possible from this condition given a specific utility function.
} 
$\chi(\delta) \equiv \delta$ (i.e., under the relative conception of discounting). Since welfare is expressed in equally distributed equivalent utility, the optimal equilibrium is not sensitive to the discount rate.

$\chi(\delta) \equiv 1$ (i.e., under the absolute conception of discounting). Since welfare is expressed in present value utility, a marginal increase in the discount rate $\delta$ leads to a lower guaranteed consumption threshold and a higher resource-legacy threshold.

This result shows that the choice between the two conceptions of discounting matters a lot when one performs comparative statics with respect to the discount rate. The intuition behind Result 7 is as follows. In this type of right-based development path with constant consumption, the minimal consumption threshold contributes both to rights and welfare in the RWI. If welfare is defined such that constant consumption streams are neutral in terms of welfare when the discount rate changes (i.e., $\chi(\delta) \equiv \delta$ ), a marginal change in the discount rate will not affect the optimal minimal rights. On the contrary, if welfare is defined in terms of present value (i.e., $\chi(\delta) \equiv 1$ ), only the utility of the present generation is welfare neutral to changes in the discount rate. A change in the discount rate influences the welfare level of a constant consumption path. The higher the discount rate, the lower the welfare value of the path. The importance of consumption diminishes when society discounts the future consumption stream more heavily. This favors the resource conservation legacy constraint in the trade-off between the two rights.

Result 8 (Trade-off between rights and welfare) For a right-based development path, a small increase in the weight of rights $\theta$ leads to a lower consumption threshold and a higher resource-legacy threshold.

The intuition behind this result is somewhat similar to that of Result 7 . If the weight of rights increases at the expense of the weight on welfare, the relative contribution of the guaranteed consumption relative to that of the resource preservation diminishes: On the one hand the weight of the minimal consumption increases as the weight of rights increases, but its contribution to welfare decreases, so that the effect on its overall contribution to the RWI is ambiguous. This favors the resource conservation threshold.

Result 9 (Trade-off among rights) For a right-based development path, an increase in the weight of the legacy constraint $\eta_{S}$ will reduce the optimal consumption threshold $\mu_{c}^{\star}$, whereas an increase in the weight of the minimum consumption $\eta_{c}$ will increase the optimal consumption threshold $\mu_{c}^{\star}$.

These last results are intuitive. 
Interior solution: Constrained utilitarian path. We now turn to the case where the optimal choice $\left(\mu_{S}^{\star}, \mu_{c}^{\star}\right)$ is neither on the threshold possibility frontier nor on the lower boundary lines $\mu_{S}=0$ or $\mu_{c}=0$. The optimal trajectory of this case is very difficult to determine, as discussed in the direct approach below. As marginal utility at zero consumption is infinite, consumption is positive at all times, and the part of the stock not set aside for preservation, $S_{0}-\mu_{S}^{\star}$, is depleted asymptotically.

The optimal thresholds must solve

$$
\max _{\mu_{c}, \mu_{S}} J\left(\mu_{c}, \mu_{S}\right) \equiv(1-\theta) V\left(S_{0}-\mu_{S}, K_{0}, \mu_{c}\right)+\theta\left(\eta_{S} \mu_{S}+\eta_{c} \mu_{c}\right)
$$

subject to $\mu_{c} \geq 0, \mu_{S} \geq 0$ and (24).

The value function $V\left(S_{0}-\mu_{S}, K_{0}, \mu_{c}\right)$ can, in principle, be computed, and for an interior solution the optimal thresholds $\mu_{c}^{\star}$ and $\mu_{S}^{\star}$ satisfy, for $i=c, S$ :

$$
\frac{\partial J}{\partial \mu_{i}}=0 \Leftrightarrow \underbrace{(1-\theta) \frac{\partial V\left(S_{0}-\mu_{S}^{\star}, K_{0}, \mu_{c}^{\star}\right)}{\partial \mu_{i}}}_{\begin{array}{c}
\text { Net present value loss from } \\
\text { increasing the minimal right }
\end{array}}+\underbrace{\theta \mathcal{R}_{\mu_{i}}^{\prime}\left(\mu_{c}^{\star}, \mu_{S}^{\star}\right)}_{\begin{array}{c}
\text { Gain in terms of } \\
\text { minimal right }
\end{array}}=0
$$

which is equivalent to $\frac{\partial}{\partial \mu_{i}}\left[V\left(S_{0}-\mu_{S}^{\star}, K_{0}, \mu_{c}^{\star}\right)\right]=-\frac{\theta}{(1-\theta)} \eta_{i}$, for $i=c, S$. We cannot characterize further the expression of $\mu_{S}^{\star}$ and $\mu_{c}^{\star}$ without knowing more details about the second order cross derivatives of the value function. ${ }^{16}$ We can say, however, that there is a unique solution, as the value function is monotonic increasing and concave in the state variable, given that the utility function is strictly increasing and concave in the consumption (for a proof, see Long, 1979). Note that, in general, extreme solutions cannot be excluded. For example, if $V_{S}^{\prime}\left(S_{0}, K_{0}, \mu_{c}\right) \geq \frac{\theta}{(1-\theta)} \eta_{S}$, it is optimal to preserve none of the resource stock, i.e., $\mu_{S}^{\star}=0$.

\subsection{The direct approach}

We now turn to the direct maximization of criterion (23). Using the same notations as in the general case for co-state variables and constraint multipliers, the current value Hamiltonian is $H^{c}=\delta \theta\left(\eta_{c} \mu_{c}+\eta_{S} \mu_{S}\right)+(1-\theta) \chi(\delta) U(c)+\psi_{K}\left[K^{\alpha} r^{\beta}-c\right]-\psi_{S} r$. The associated Lagragian is $L^{c}=H^{c}+w_{c}\left(c-\mu_{c}\right)+w_{S}\left(S-\mu_{S}\right)+\nu_{c} c+\nu_{r} r+\nu_{K} K+\nu_{S} S$, where

\footnotetext{
${ }^{16}$ It is usually not possible to have a closed-form solution to problem (25), except under some restrictive conditions (Benchekroun and Withagen, 2011).
} 
the $\nu$ are the multipliers of non-negativity constraints for state and control variables. ${ }^{17}$ The necessary conditions, given in the appendix, lead to the following results.

Result 10 (Positive minimal consumption and egalitarian tail) Assume $\eta_{c}>0$. Then the optimal consumption threshold $\mu_{c}^{\star}$ is strictly positive, and there is a finite time $T$ such that, for all $t>T, c(t)=\mu_{c}^{\star}$.

In the DHS model, along the unconstrained utilitarian path, consumption is always strictly positive, approaching zero only asymptotically (Dasgupta and Heal, 1974). Therefore, raising $\mu_{c}$ infinitesimally above zero will have effects only on the consumption in the far future, and thus, since $\delta>0$, will have negligible marginal effect on welfare. ${ }^{18}$ Yet, the marginal effect of the minimum consumption on the right index is $\eta_{c}>0$. It follows that it is optimal to raise $\mu_{c}$ above zero, i.e. $\mu_{c}^{\star}>0$.

Result 11 (Efficient resource use and resource value) If the excess of the initial resource stock over the preserved stock, $S(0)-\mu_{S}^{\star}$, is stricly positive, it will be asymptotically depleted, with $S(t)>\mu_{S}^{\star}$ at all finite times $t$. The resource extraction is efficient and satisfies Hotelling's rule, i.e., $\frac{1}{F_{r}^{\prime}} \frac{d F_{r}^{\prime}}{d t}=F_{K}^{\prime}$. If $\mu_{S}^{\star}>0$, the initial resource shadow value given by $\psi_{S}(0)=\theta \eta_{S}{ }^{19}$

The introduction of the optimal rights does not interfere with the dynamic efficiency condition given by Hotelling's rule, but in general it influences the resource price, possibly resulting in a preservation of a part of the resource stock forever.

Result 12 (Minimal consumption and discounting) Once the minimal consumption level is reached, the consumption discount rate has to differ from the return on capital to account for the shadow cost of satisfying the minimal consumption right $\left(w_{c}\right)$. One has $F_{K}^{\prime}=\delta-\frac{\dot{w}_{c}}{(1-\theta) U^{\prime}\left(\mu_{c}^{\star}\right)+w_{c}}$, with $\dot{w}_{c}<0$ when $F_{K}^{\prime}>\delta$ and $\dot{w}_{c}>0$ when $F_{K}^{\prime}<\delta$.

This result corresponds to a modified Keynes-Ramsey rule. Along any consumption path, the consumption discount rate is $\rho(t)=\delta-\frac{\dot{U}^{\prime}}{U^{\prime}}$, which is usually expressed as the sum of utility discount rate and the wealth effect: $\rho(t)=\delta+\frac{1}{\sigma} \frac{\dot{c}}{c}$, where $\sigma \equiv \frac{-U^{\prime}}{c U^{\prime \prime}}$ is the elasticity of intertemporal substitution. In a competitive economy without externalities and policy intervention, the condition for optimal intertemporal consumption allocation implies that

\footnotetext{
${ }^{17}$ The multipliers associated with the constraints $\mu_{c} \geq 0$ and $\mu_{S} \geq 0$ will appear in separate conditions (see eqs. (39) and (40) in Appendix A.3).

${ }^{18}$ Notice that $\mu_{S}$ can be kept constant when $\mu_{c}$ is raised marginally from zero: only the consumption path has to be marginally modified.

${ }^{19}$ If $\mu_{S}^{\star}=0$, then $\psi_{S}(0) \geq \theta \eta_{S}$. We thank the editor in charge for pointing this out.
} 
the consumption discount rate equals the return on investment, i.e., $\rho(t)=F_{K}^{\prime}$. One can then derive the usual expression of the Keynes-Ramsey rule from the previous expression: $\frac{\dot{c}}{c}=\sigma\left(F_{K}^{\prime}-\delta\right)$. Along a constant consumption path, the rate of change of marginal utility is nil (there is no wealth effect), and the non-arbitrage condition implies that the consumption discount rate equals the utility discount rate $\delta$. When the minimum consumption constraint is binding such that $c=\mu_{c}^{\star}>0$ and $w_{c}>0$, this condition is modified (see eqs. 35 and 37 in the Appendix).

It follows that, if the RWI allocation is to be achieved by a decentralized mechanism, consumers should be facing a constant interest rate at level $\delta$, so that constant consumption is intertemporally efficient, in spite of the fact that the return on capital is decreasing as capital accumulates. Then, in the decentralized implementation, there must be a wedge between the consumer's interest rate and the producer's rental rate. This wedge between the producer's and consumer's interest rates implies tax or subsidy on savings, to ensure minimal consumption rights. The wedge will be negative when $F_{K}^{\prime}$ is greater than $\delta$, i.e., when the capital stock is low at the earlier stage of development of the economy, and positive after some time, as $F_{K}^{\prime}$ converges to zero when the capital stock tends to infinity. ${ }^{20}$

\section{Discussion and concluding remarks}

How to be moral in a pragmatic world? How to reconcile ethical norms with selfish interests? The present paper introduces a criterion that accounts for rights and welfare in ranking social alternatives of development paths. The criterion is a weighted sum of an index of minimal rights guaranteed to all generations and a welfare index. Such a criterion could represent the development of a society that collectively defines minimal rights to be guaranteed over time (e.g., rights related to the environmental quality) while individuals make their own private decisions (e.g., on consumption and investment) maximizing wellbeing. These rights are implemented by the social planner as collective constraints. Such collective constraints, when defined in the context of environmental issues such as climate change or biodiversity erosion, could represent the objectives of a sustainable development.

We show that the definition of minimal rights should account for both the tension between rights and welfare, i.e., the welfare cost of sustaining the minimal rights, and for the trade-offs among rights. These two dimensions create an opportunity cost for each

\footnotetext{
${ }^{20}$ That marginal product of capital is high earlier in the program, and later falls to zero, is due both to the increase in the capital stock and the decrease in extraction, as shown in, e.g., Dasgupta and Heal (1979) and Leonard and Long (1991, Chapter 9).
} 
right, which is the present value of the (shadow) cost of complying with the corresponding constraint over time. Accounting for the induced costs, both in terms of forgone welfare and conflicting rights, calls for a balanced setting of the level of each right. Not all levels of rights are feasible and compatible with one another. Taken in isolation, it would be tempting to increase the levels of some rights at some welfare cost. This may, however, not be done due to conflicts with other sustainability issues and corresponding rights. As a consequence, a right may not be granted at a level maximizing the associated moral value. By accounting for rights and welfare in a mixed criterion, the RWI criterion introduces consistency in the definition of minimal rights.

We illustrate the general results in the canonical model of production-consumption with nonrenewable resource developed after Dasgupta and Heal $(1974,1979)$ and Solow (1974). Our example highlights the possibility that, at some point, minimal rights may be so important that the willingness to satisfy these minimal rights drives the development path (right-based sustainable development). The development trajectory may strongly differ from the competitive unconstrained path. In particular, if sustaining a positive consumption level is desired, one has to modify the Keynes-Ramsey rule to smooth consumption over time and adjust investment. This can be done by influencing the discount rate the consumers face, and make it different from the producers' discount rate as defined by the marginal productivity of capital. This may imply some wedge between consumers and producers interest rates, possibly implemented by tax or subsidy on savings. These results have important implications in the definition of the discount rate to be applied on investment projects, as they are discussed in the climate change mitigation debate.

It is important to acknowledge that the proposed criterion is likely to result in time-inconsistent solutions. Mixed criteria proposed by Chichilnisky (1996) or AlvarezCuadrado and Long (2009) have the property that in general the optimal solution is timeinconsistent. As time goes by, the utilities in the distant future, which were negligible at the time the plan was conceived, become important, and their weights in the trade-off (against minimum utility, as in Alvarez-Cuadrado and Long, or against the golden rule utility, as in Chichilnisky) are no longer negligible. Optimizing the criterion again would result in a different solution. Time-inconsistency can also emerge without discounting, as in Martinet (2011). The definition of minimal rights to be guaranteed forever depends on the possibilities offered by the state of the economy. As the capital stocks evolve, some opportunities are lost (e.g., on the preservation of nonrenewable resources once depleted) whereas other opportunities become available (e.g., on the level of sustainable consumption as manufactured capital accumulates). Minimal rights would also be revised over time, notwithstanding welfarist considerations. The RWI proposed in this paper shares 
both sources of time-inconsistency. First, the set of achievable minimal rights depends on the current resources endowment and evolves over time. Second, the welfare cost of a right will also evolve over time, as the periods at which the associated constraints are binding influence the opportunity cost of rights.

Even if time-inconsistency seems to be a strong limitation, it is now well-established in the literature that time-consistency is more likely to be an exception more than the rule in many dynamic social choice problems. Jackson and Yariv (2015) show that, even when individuals have preferences represented by constant discounted utility, a non-dictatorial collective decision rule has to be time-inconsistent. It would be welcome to have a general picture of compatibilities and incompatibilities between intergenerational equity conditions and time consistency. Recently, Asheim and Mitra (2017) have proposed such an analysis. Interpreting the RWI criterion as the weighted sum of the preferences of proponents of rights (maximin-like agents) and proponents of welfare (discounted utility-like agents), time-inconsistency seems inevitable. Searching for a time-consistent criterion with good equity properties is likely to be unsuccessful. A better option would be to think about ways to either implement time-consistent paths (for example, with commitment) or to analyze the consequences of the revision of objectives. On the one hand, the current decision maker would be naive to set targets without accounting for the fact that they may be revised. On the other hand, time-inconsistency may be an issue only retrospectively, and a forward-looking society may overlook it. If time-inconsistency is a problem, or a source of inefficiency, it is so only from the point of view of current generation/decision-maker, which would not be in charge when the objectives and development plans are revised. For future generations/decision-makers, time-inconsistency means improvement, thanks to new opportunities in addition to the still feasible previously chosen objectives. A challenge for future research is thus to define sustainability criteria that account for the opportunities bequeathed to future generations more than for the currently estimated value of particular development paths.

\section{A Appendix}

\section{A.1 Proof of Proposition 1}

The optimization problem (7) is equivalent to

$$
V\left(x_{0} ; \mu_{1}, \ldots, \mu_{I}\right)=\max _{(x(\cdot), c(\cdot)) \in \mathcal{F}\left(x_{0} ; \mu_{1}, \ldots, \mu_{I}\right)} \mathcal{W}(x(\cdot), c(\cdot))
$$


For any set of thresholds $\left(\mu_{1}, \ldots, \mu_{I}\right)$ and $\left(\mu_{1}^{\prime}, \ldots, \mu_{I}^{\prime}\right)$ such that $\mu_{i}^{\prime} \geq \mu_{i}$ for $i=1, \ldots, I$, we have $\mathcal{F}\left(x_{0} ; \mu_{1}^{\prime}, \ldots, \mu_{I}^{\prime}\right) \subseteq \mathcal{F}\left(x_{0} ; \mu_{1}, \ldots, \mu_{I}\right)$. This implies that $V\left(x_{0} ; \mu_{1}^{\prime}, \ldots, \mu_{I}^{\prime}\right) \leq$ $V\left(x_{0} ; \mu_{1}, \ldots, \mu_{I}\right)$.

\section{A.2 Direct approach to RWI maximization: Mathematical de- tails}

The necessary conditions The first-order necessary conditions of the optimization problem are as follows. ${ }^{21}$

The control variables maximize the Lagrangean, i.e., $\frac{\partial L^{c}}{\partial c}=(1-\theta) \chi(\delta) U_{c}^{\prime}+\psi g_{c}^{\prime}+$ $\Delta h_{c}^{\prime}+w \mathcal{I}_{c}^{\prime}=0$. The shadow-values satisfy $\dot{\psi}=\delta \psi-\frac{\partial L^{c}}{\partial x}$. The stock dynamics imply $\dot{x}=\frac{\partial L^{c}}{\partial \psi}$. The satisfaction of the admissibility constraints implies $\Delta \geq$ $0, \quad h(x, c) \geq 0, \quad \Delta h(x, c)=0$. The satisfaction of the right-based constraints implies $w \geq 0, \quad \mathcal{I}(x, c)-\mu \geq 0, \quad w[\mathcal{I}(x, c)-\mu]=0$. Moreover, when pure state constraints (Eqs. 14) are imposed for all $t$, technically this implies specifying a 'terminal manifold' for the associated state variables: $\lim _{t \rightarrow \infty} x_{i}(t) \geq \mu_{i}, \quad i=1,2, \ldots, p$. The transversality conditions with respect to these stocks are $\lim _{t \rightarrow \infty} \pi_{i}(t) \geq 0$ and $\lim _{t \rightarrow \infty} \pi_{i}(t)\left(x_{i}(t)-\mu_{i}\right)=0$.

The optimality conditions with respect to the choice of the control parameters $\mu_{i} \geq 0$ are

$$
\begin{aligned}
\int_{0}^{\infty} e^{-\delta t} \frac{\partial L^{c}}{\partial \mu_{i}} d t=\bar{\pi}_{i}, \quad i & =1,2, \ldots, p \\
\int_{0}^{\infty} e^{-\delta t} \frac{\partial L^{c}}{\partial \mu_{i}} d t & =0, \quad i=p+1, p+2, \ldots, I
\end{aligned}
$$

with $\bar{\pi}_{i} \equiv \lim _{t \rightarrow \infty} \pi_{i}(t)$.

From Eqs. (12) and (13), for all $i$ we have $\frac{\partial L^{c}}{\partial \mu_{i}}=\delta \theta \mathcal{R}_{\mu_{i}}^{\prime}\left(\mu^{\star}\right)-w_{i}$. As $\mathcal{R}$ does not depend on time, neither do its derivatives. The optimality conditions (30) and (31) therefore become

$$
\begin{aligned}
& \theta \mathcal{R}_{\mu_{i}}^{\prime}-\int_{0}^{\infty} e^{-\delta t} w_{i}(t) d t=\bar{\pi}_{i}, \quad i=1, \ldots, p \\
& \theta \mathcal{R}_{\mu_{i}}^{\prime}-\int_{0}^{\infty} e^{-\delta t} w_{i}(t) d t=0, \quad i=p+1, \ldots, I
\end{aligned}
$$

If a legacy constraint is binding in the limiting sense only, $w_{i}(t)=0$ at all times and

\footnotetext{
${ }^{21}$ We here consider the first order, necessary conditions only, for the sake of simplicity. The sufficiency conditions (concavity conditions) can be derived as in Leonard and Long (1991). We also assume that constraint qualifications are satisfied (see Takayama, 1985).
} 
eq. (32) becomes $\theta \mathcal{R}_{\mu_{i}}^{\prime}=\bar{\pi}_{i}$.

For any $i$ such that $\mathcal{R}_{\mu_{i}}^{\prime}>0$, this implies that either $w_{i}(t)>0$ on some time interval, or $\lim _{t \rightarrow \infty} e^{-\delta t} \psi_{i}(t)>0$. The associated constraint $\mathcal{I}_{i}(x, c) \geq \mu_{i}$ is binding, either on an interval for $i=p+1, \ldots, I$, or in the limiting sense for $i=1, \ldots, p$.

\section{A.3 DHS model: Mathematical details}

Indirect approach: Proofs of the comparative static results for right-based development paths

Proof of Result 7: Since the optimality condition (27) holds as an identity, we can differentiate both sides with respect to $\delta$, treating $\mu_{c}^{\star}$ as an implicit function of $\delta$.

For $\chi(\delta) \equiv \delta$, the identity does not depend on $\delta$ and thus $\frac{\partial \mu_{c}^{\star}}{\partial \delta}=0$.

For $\chi(\delta) \equiv 1$, we obtain $\frac{\partial \mu_{c}^{\star}}{\partial \delta}=\frac{1}{G} \frac{(1-\theta) \delta^{-2} U^{\prime}}{\theta \eta_{S}}<0$ where $G \equiv \frac{(1-\theta) \delta^{-1} U^{\prime \prime}}{\theta \eta_{S}}+\bar{\mu}_{S}^{\prime \prime}\left(\mu_{c}^{\star}\right)<0$.

Proof of Result 8: Re-write the optimality condition (27) as $\frac{(1-\theta) \delta^{-1} U^{\prime}\left(\mu_{c}^{\star}\right)}{\theta \eta_{S}}+\frac{\eta_{c}}{\eta_{S}}=$ $-\bar{\mu}_{S}^{\prime}\left(\mu_{c}^{\star}\right)$. Then $\frac{\partial \mu_{c}^{\star}}{\partial \theta}=-\frac{1}{G} \delta^{-1} U^{\prime}\left(\mu_{c}^{\star}\right) \frac{d}{d \theta}\left(\frac{1-\theta}{\theta}\right)<0$.

The proof of Result 9 is straightforward, and is therefore omitted.

Indirect approach: Characterization of the constrained utilitarian paths Since $U^{\prime}(0)=\infty$, consumption is never nil. Suppose there is some $\mu_{S}>0$ that is set aside "from the beginning." To determine $\mu_{S}$ and $\mu_{c}$ we can follow a two-step procedure.

Step 1: Consider the discounted utility maximization à la Dasgupta and Heal, coupled with a minimum consumption constraint, $c(t) \geq \mu_{c}$. This gives rise to an associated value function for an initial stock of resource $S_{0}-\mu_{S}$ :

$$
\begin{aligned}
& V\left(S_{0}-\mu_{S}, K_{0}, \mu_{c}\right) \equiv \max _{c, r} \int_{0}^{\infty} e^{-\delta t} U(c(t)) d t \\
& \text { s.t. } \quad \dot{K}=K(t)^{\alpha} r(t)^{\beta}-c(t), K(0)=K_{0}, K(t) \geq 0, \\
& \dot{S}=-r(t), S(0)=S_{0}, \lim _{t \rightarrow \infty} S(t)=\mu_{S}, \\
& c(t) \geq \mu_{c} .
\end{aligned}
$$

This function can in principle be calculated (though not in closed form). ${ }^{22}$

Step 2: Choose $\mu_{c} \geq 0$ and $\mu_{S} \geq 0$ to maximize

$$
(1-\theta) V\left(S_{0}-\mu_{S}, K_{0}, \mu_{c}\right)+\theta\left(\eta_{c} \mu_{c}+\eta_{S} \mu_{s}\right)
$$

\footnotetext{
${ }^{22}$ For some special cases of problem (34), it is possible to obtain a closed form solution for the value function. In this case, using the expression of the value function, it is possible to solve explicitly problem $(28)$.
} 
subject to $\phi\left(\mu_{c}, \mu_{S}, K_{0}, S_{0}\right) \geq 0$. Since the function $V\left(S_{0}-\mu_{S}, K_{0}, \mu_{c}\right)$ is not analytically tractable, one will have to rely on numerical solutions.

Direct approach: Necessary conditions The necessary conditions for the maximization of the Lagrangian are

$$
\begin{aligned}
\frac{\partial L^{c}}{\partial c}=0 & \Leftrightarrow \quad(1-\theta) \chi(\delta) U^{\prime}-\psi_{K}+w_{c}+\nu_{c}=(835) \\
\frac{\partial L^{c}}{\partial r}=0 & \Leftrightarrow \quad \psi_{S}=\psi_{K} F_{r}^{\prime}+\nu_{r} \\
\dot{\psi}_{K}=\delta \psi_{K}-\frac{\partial L^{c}}{\partial K} & \Leftrightarrow \quad \dot{\psi}_{K}=\psi_{K}\left(\delta-F_{K}^{\prime}\right)-\nu_{K} \\
\dot{\psi}_{S}=\delta \psi_{S}-\frac{\partial L^{c}}{\partial S} & \Leftrightarrow \quad \dot{\psi}_{S}=\delta \psi_{S}-w_{S}-\nu_{S} \\
\xi_{c}+\int_{0}^{\infty} e^{-\delta t} \frac{\partial L^{c}}{\partial \mu_{c}} d t=0 & \Leftrightarrow \xi_{c}+\quad \theta \eta_{c}-\int_{0}^{\infty} e^{-\delta t} w_{c} d t=0 \\
\xi_{S}+\int_{0}^{\infty} e^{-\delta t} \frac{\partial L^{c}}{\partial \mu_{S}} d t=\lim _{t \rightarrow \infty} e^{-\delta t} \psi_{S}(t) \geq 0 & \Leftrightarrow \xi_{S}+\quad \theta \eta_{S}-\int_{0}^{\infty} e^{-\delta t} w_{S} d t=\bar{\pi}_{S} \geq 0
\end{aligned}
$$

with $\xi_{c} \geq 0, \mu_{c} \geq 0, \xi_{c} \mu_{c}=0, \xi_{S} \geq 0, \mu_{S} \geq 0, \xi_{S} \mu_{S}=0, \bar{\pi}_{S}=\lim _{t \rightarrow \infty} e^{-\delta t} \psi_{S}(t)$ and $w_{c} \geq 0, w_{c}\left(c-\mu_{c}\right)=0$, and $w_{S} \geq 0, w_{S}\left(S-\mu_{S}\right)=0$, as well as $\nu_{x} \geq 0, x \geq 0$ and $\nu_{x} x=0$ for $x=c, r, K, S$.

We can first state that $\left(\nu_{c}, \nu_{r}, \nu_{K}, \nu_{S}\right)=(0,0,0,0)$, and thus that the non-negativity constraints are never binding along the optimal path. Given that $\lim _{c \rightarrow 0} U^{\prime}(c)=+\infty$, consumption would be strictly positive whenever possible. Exhausting the initial capital stock in finite time would thus be inefficient, and $K(t)>0$ at all times, implying $\nu_{K}=0$. A nil extraction would yield an infinite marginal productivity of the resource, which is not compatible with eq. (36). Thus, $r(t)>0$ and $\nu_{r}=0$. Positive extraction at all finite times $t$ implies that resource must not be exhausted in finite time, thus $S(t)>0$ at all times, implying $\nu_{S}=0$. Given positive extraction and production, a positive consumption is feasible at all times, and since $U^{\prime}(0)=\infty$, we have $c(t)>0$ and $\nu_{c}=0$. In what follows, we refer to the previous first order conditions with $\left(\nu_{c}, \nu_{r}, \nu_{K}, \nu_{S}\right)=(0,0,0,0)$.

Eq. (35) characterizes the capital shadow value as being the marginal utility weighted by the share of welfare in the RWI, plus the cost of the minimal consumption constraint. Eq. (36) characterizes the natural resource shadow value as being the marginal productivity of the resource times the capital shadow value. Eqs. (37) and (38) are related to Keynes-Ramsey's rule and Hotelling's rule. Eq. (39) and (40) correspond to the optimality 
conditions on minimal rights.

From eq. (39), we deduce that $\int_{0}^{\infty} e^{-\delta t} w_{c} d t>0$, which means that the constraint $c(t) \geq \mu_{c}$ is binding after some time. This constitutes the proof of Result $\mathbf{1 0}$.

In the long-run, a positive amount of resource must be extracted to meet the positive consumption constraint $c(t) \geq \mu_{c}^{\star}>0$. The resource constraint is thus never binding, and therefore $w_{S}(t)=0$ for all $t$. We obtain $\dot{\psi}_{S}=\delta \psi_{S}$ from eq. (38), which implies $\frac{\dot{\psi}_{S}}{\psi_{S}}=\delta$. From eq. (36), we obtain $\frac{\dot{\psi}_{S}}{\psi_{S}}=\frac{\dot{\psi}_{K}}{\psi_{K}}+\frac{d \ln \left(F_{r}^{\prime}\right)}{d t}$. Substituting $\frac{\dot{\psi}_{K}}{\psi_{K}}=\delta-F_{K}^{\prime}$ from eq. (37) in this expression and equating the two expression for $\frac{\dot{\psi}_{S}}{\psi_{S}}$, we obtain $\frac{d \ln \left(F_{r}^{\prime}\right)}{d t}=F_{K}^{\prime}$, which is Hotelling's rule. Moreover, the integral part in condition (40) is equal to zero along the optimal path, and $\lim _{t \rightarrow \infty} e^{-\delta t} \psi_{S}(t)=\psi_{S}(0)$. Then, condition (40) reduces to $\bar{\pi}_{S} \equiv \psi_{S}(0)=\theta \eta_{S}$ if $\mu_{S}^{\star}>0$. (If $\mu_{S}^{\star}=0$, then $\bar{\pi}_{S} \equiv \psi_{S}(0) \geq \theta \eta_{S}$.) This completes the Proof of Result 11.

Consider now the constant consumption part of the path, with $c(t)=\mu_{c}^{\star}$. Taking the time derivative of eq. (35), we obtain $(1-\theta) \dot{U}^{\prime}=\dot{\psi}_{K}-\dot{w}_{c}$. As consumption is constant, this expression is nil and we obtain $\dot{\psi}_{K}=\dot{w}_{c}$ on this part of the path. Moreover, using eq. (37), we have $F_{K}^{\prime}=\delta-\frac{\dot{\psi}_{K}}{\psi_{K}}$, which, combined with the previous equality and with eq. (35) gives $F_{K}^{\prime}=\delta-\frac{\dot{w}_{c}}{(1-\theta) U^{\prime}\left(\mu_{c}^{\star}\right)+w_{c}}$. This completes the Proof of Result 12.

\section{References}

Alvarez-Cuadrado F. and Long N.V. (2009) A mixed Bentham-Rawls criterion for intergenerational equity: Theory and implications. Journal of Environmental Economics and Management 58:154-168.

Asheim G.B. and Ekeland I. (2016) Resource conservation across generations in a RamseyChichilnisky model. Economic Theory 61:611-639.

Asheim G.B. and Mitra T. (2017) The necessity of time inconsistency for intergenerational equity, Mimeo, University of Oslo, January 2017.

Asheim G.B. and Zuber S. (2013) A complete and strongly anonymous leximin relation on infinite streams. Social Choice and Welfare 41:819-834.

Benchekroun H. and Withagen C. (2011) The optimal depletion of exhaustible resources: A complete characterization. Resource and Energy Economics, 33(3):612-636.

Burmeister E. and Hammond P.J. (1977) Maximin paths of heterogeneous capital accumulation and the instability of paradoxical steady states. Econometrica 45:853-870. 
Cairns R. and Long N.V. (2006) Maximin, a direct approach to sustainability. Environmental and Development Economics 11:275-300.

Chakravorty U., Moreaux M. and Tidball M. (2008) Ordering the extraction of polluting nonrenewable resources. American Economic Review 98(3):1128-1144.

Chichilnisky G. (1996) An Axiomatic Approach to Sustainable Development. Social Choice and Welfare 13:231-257.

Chichilnisky G., Heal G. and Beltratti A.(1995) The Green Golden Rule. Economics Letters 49(2):175-179.

Dasgupta P. and Heal G. (1974) The Optimal Depletion of Exhaustible Resources. Review of Economic Studies 41:1-28.

Dasgupta P. and Heal G. (1979) Economic Theory and Exhaustible Resources. Cambridge University Press.

D'Autume A., Hartwick J. and Schubert K. (2010) The zero discounting and maximin optimal paths in a simple model of global warming. Mathematical Social Sciences, 59:193-207.

Deb R. (1994) Waiver, Effectivity, and Rights as Game Forms. Economica 61:167-178.

Figuieres C., Long N.V. and Tidball M. (2017) The Mixed Bentham-Rawls Criterion of Intertemporal Choice Criterion and Rawls' Just Savings Principle. Mathematical Social Sciences 85:11-22.

Gaertner W., Pattanaik P.K. and Suzumura K. (1992) Individual Rights Revisited. Economica 59:161-177.

Gärdenfors P. (1981) Rights, Games and Social Choice. Noûs 15:341-356.

Hammond P.J. (1995) Social Choice of Individual and Group Rights. In Barnett W., Moulin H., Salles M. and Schofield N. (eds.), Social Choice, Welfare, and Ethics, Cambridge: Cambridge University Press, pp.55-77.

Hammond P.J. (1996) Game Forms versus Social Choice Rules as Models of Rights. In Arrow K.J., Sen A.K. and Suzumura K. (eds.), Social Choice Re-examined, Vol.2, London: Macmillan, pp.82-95. 
Heal G. (1998) Valuing the Future. Economic Theory and Sustainability. Columbia University Press, New York.

Harstad B. (2012) Buy Coal! A Case for Supply-Side Environmental Policy. Journal of Political Economy, 120(1):77-115.

Howarth R. (1995) Sustainability under uncertainty: A deontological approach. Land Economics, 71(4):417-427.

Jackson M. and Yariv L. (2015) Collective Dynamic Choice: The necessity of time inconsistency. American Economic Journal: Microeconomics, 7(4):150-78

Koopmans T. (1960) Stationary ordinal utility and impatience. Econometrica, 28(2):287309.

Lauwers L. (1997a) Rawlsian equity and generalised utilitarianism with an infinite population. Economic Theory, 9(1):143-150.

Lauwers L. (1997b) Continuity and equity with infinite horizons. Social Choice and Welfare, 14(2):345-356.

Lauwers L. (1998) Intertemporal objective functions: Strong pareto versus anonymity, Mathematical Social Sciences, 35(1):37-55.

Leonard D. and Long N.V. (1991) Optimal Control Theory and Static Optimization in Economics, Cambridge University Press, Cambridge.

Long N.V. (1979) Two Theorems on Generalized Diminishing Returns and their Applications to Economic Analysis. Economic Record, 55:58-63.

Long N.V. (2007) Toward a Theory of a Just Savings Principle. In J. Roemer and K. Suzumura (Eds.), Intergenerational Equity and Sustainability, Palgrave, London.

Long N.V. (2011) A Dynamic Game of Environmental Exploitation between Two Countries with Sequential Maximin Objectives. International Journal of Development and Conflict 1(3):1-15.

Martinet V. (2011) A Characterization of Sustainability with Indicators. Journal of Environmental Economics and Management 61:183-197.

Martinet V. (2012) Economic Theory and Sustainable Development. What Can we preserve for future generations?, Routledge, London and New York. 
Martinet V. and Doyen L. (2007) Sustainability of an economy with an exhaustible resource: A viable control approach. Resource and Energy Economics 29:17-39.

Neumayer E. (2013) Weak Versus Strong Sustainability: Exploring the Limits of Two Opposing Paradigms. Edward Elgar Publishing.

Nozick R. (1974) Anarchy, State and Utopia, New York: Basic Books.

Peleg B. (1998) Effectivity Functions, Game Forms, Games, and Rights. Social Choice and Welfare 15:67-80.

Rawls J. (1971) A theory of Justice. Oxford, England: Clarendon.

Reiss H. (1970) Kant's Political Writings. Cambridge University Press, Cambridge.

Sen A.K. (1970a) Collective Choice and Social Welfare, San Francisco: Holden-Day. Republished, Amsterdam: North-Holland, 1979.

Sen A.K. (1970b) The Impossibility of a Paretian Liberal. Journal of Political Economy $78: 152-157$.

Sen A.K. (1976) Liberty, Unanimity and Rights. Economica 43:217-241.

Solow R.M. (1974) Intergenerational Equity and Exhaustible Resources. Review of Economic Studies 41:29-45.

Stollery K. (1998) Constant utility paths and irreversible global warming. Canadian Journal of Economics, 31(3):730-742.

Sugden R. (1985) Liberty, Preference and Choice. Economics and Philosophy 1:213-229.

Suzumura K. (2005) An interview with Paul Samuelson: welfare economics, "old" and "new" and social choice theory. Social Choice and Welfare 25(2):327-356.

Suzumura K. and Yoshihara N. (2008) On Initial Conferment of Individual Rights. Working Paper, Institute of Economic Research, Hitotsubashi University.

Takayama A. (1985) Mathematical Economics (2nd edition). Cambridge University Press.

Tol R.S.J. (2013) Climate policy with Bentham-Rawls preferences. Economics Letters 118(3): 424-428.

World Commission on Environment and Development - WCED (1987) Our common Future, Oxford University Press. 